\title{
One TPM to Bind Them All: Fixing TPM 2.0 for Provably Secure Anonymous Attestation
}

\author{
Jan Camenisch*, Liqun Chen ${ }^{\dagger}$, Manu Drijvers* ${ }^{*}$, Anja Lehmann*, David Novick ${ }^{\S}$, and Rainer Urian \\ ${ }^{*}$ IBM Research - Zurich, ${ }^{\dagger}$ University of Surrey, ${ }^{\ddagger}$ ETH Zurich, ${ }^{\S}$ Intel, ${ }^{\uparrow}$ Infineon
}

\begin{abstract}
The Trusted Platform Module (TPM) is an international standard for a security chip that can be used for the management of cryptographic keys and for remote attestation. The specification of the most recent TPM 2.0 interfaces for direct anonymous attestation unfortunately has a number of severe shortcomings. First of all, they do not allow for security proofs (indeed, the published proofs are incorrect). Second, they provide a Diffie-Hellman oracle w.r.t. the secret key of the TPM, weakening the security and preventing forward anonymity of attestations. Fixes to these problems have been proposed, but they create new issues: they enable a fraudulent TPM to encode information into an attestation signature, which could be used to break anonymity or to leak the secret key. Furthermore, all proposed ways to remove the Diffie-Hellman oracle either strongly limit the functionality of the TPM or would require significant changes to the TPM 2.0 interfaces. In this paper we provide a better specification of the TPM 2.0 interfaces that addresses these problems and requires only minimal changes to the current TPM 2.0 commands. We then show how to use the revised interfaces to build $q$-SDH- and LRSW-based anonymous attestation schemes, and prove their security. We finally discuss how to obtain other schemes addressing different use cases such as key-binding for U-Prove and e-cash.
\end{abstract}

\section{INTRODUCTION}

The amount of devices connected to the Internet grows rapidly and securing these devices and our electronic infrastructure becomes increasingly difficult, in particular because a large fraction of devices cannot be managed by security professional nor can they be protected by firewalls. One approach to achieve better security is to equip these devices with a root of trust, such as a Trusted Platform Module (TPM), a Trusted Execution Environment (TEE), and Software Guard Extensions (SGX), and then have that root of trust attest to the state of the device or to computations made. When doing such attestations, it is crucial that they be privacy-protecting. On the one hand, to protect the privacy of users of such devices, and on the other hand, to minimize the information available to attackers. Realizing this, the Trusted Computing Group (TCG) has developed a protocol called direct anonymous attestation (DAA) [1] and included it in their TPM 1.2 specification [2]. The protocol allows a device to authenticate as a genuine device (i.e., that it is certified by the manufacturer) and attest to messages without the different attestations being linkable to each other and has since been implemented in millions of chips.

Later, Brickell and Li [3] proposed a scheme called Enhanced-privacy ID (EPID) that is based on elliptic curves and adds signature-based revocation which is a revocation capability based on a previous signature of a platform. This scheme has become Intel's recommendation for attestation of a trusted system, has been incorporated in Intel chipsets and processors, and is recommended by Intel to serve as the industry standard for authentication in the Internet of Things. Being based on elliptic curves, EPID is much more efficient than the original RSA-based DAA scheme. Therefore, the TCG has revised the specification of the TPM and switched to elliptic curve-based attestation schemes [4], [5]. The design idea of this new specification is rather beautiful: the TPM only executes a simple core protocol that can be extended to build different attestation schemes. Essentially, the core protocol is a Schnorr proof of knowledge of a discrete logarithm [6], the discrete logarithm being the secret key stored and protected inside the TPM. Chen and $\mathrm{Li}$ [5] describe how to extend this proof of knowledge to DAA schemes, one based on the $q$ SDH assumption [14] and one based on the LRSW assumption [15]. The idea here is that the host in which the TPM is embedded extends the protocol messages output by the TPM into messages of the DAA protocol. They further show how to extend it to realize device-bound U-Prove [7], so that the U-Prove user secret key is the one stored inside the TPM.

Unfortunately, the core protocol as specified has severe shortcomings. First, the random oracle based security proof for attestation unforgeability by Chen and $\mathrm{Li}$ is flawed [8] and indeed it seems impossible to prove that a host cannot attest to a message without involving the TPM. Second, the core protocol can be abused as a Diffie-Hellman oracle w.r.t. the secret key tsk inside the TPM. It was shown that such an oracle weakens the security, as it leaks a lot of information about tsk [26]. Further, the presence of the oracle prevents forward anonymity, as an attacker compromising a host can identify the attestations stemming from this host.

These issues were all pointed out in the literature before and fixes have been proposed [8]-[10]. However, the proposed fixes either introduce new problems or are hard to realize. Xi et al. [8] propose a change to the TPM specification that allows one to prove the unforgeability of TPM-based attestations. This change introduces a subliminal channel though, i.e., a subverted TPM could now embed information into the values it produces and thereby into the final attestation. This covert channel could be used to break anonymity of the platform and its user, or to leak the secret key held in the TPM. The proposed fixes to remove the static Diffie-Hellman oracle [8]-[10] either require substantial changes to the TPM to the extend that they are not implementable, or restrict the functionality of the TPM too much, excluding some major DAA schemes from 
being supported. For instance, it was priorly proposed to have the host prove in zero knowledge that a new base is safe to use for the TPM, who then needs to verify that proof. This does not only take a heavy toll on the resources of the TPM but also excludes signature-based revocation, thus not meeting the requirements of the TCG. We refer to Sec. 3 for a detailed discussion of the existing proposals and their shortcomings.

Our Contributions. In this paper we provide a new specification of the DAA-related interfaces of the TPM that requires only minimal changes to the current TPM 2.0 commands. It is the first one that addresses all the issues discussed and that can easily be implemented on a TPM. We then show what kind of proof of knowledge statements can be proven with the help of our new TPM interfaces and how to build secure DAA schemes with them. Our specification supports both LRSW-based and $q$-SDH-based direct anonymous attestation, signature-based revocation, and extensions to attributes. Our LRSW-based scheme has a new way to issue credentials that is much more efficient than prior ones that aimed to avoid a DH-oracle in the TPM interfaces. To achieve this, we use a slight modification of the LRSW assumption (which we prove to hold in the generic group model). Avoiding this modification would be possible, but requires a second round of communication with the issuer.

We further show how to extend the DAA schemes to support attributes and signature-based revocation and give security proofs for all of that. For space reasons, we give only sketches in this extended abstract and refer to the full version of this paper for the detailed proofs. The TPM interfaces that we give can also be used to realize other schemes, such as devicebound U-Prove [7] and e-cash [11], for which it is beneficial that a secret key be kept securely inside a TPM.

To make the construction of such schemes easier, we give for the first time a thorough characterization of statements that can be proven with a TPM w.r.t. a secret key inside the TPM. We provide a generic protocol that orchestrates our new TPM interfaces and allows one to generate TPM-based proofs for a wide class of statements. We further prove the security of such generated TPM-based proofs. This facilitates the use of the TPM interfaces for protocol designers who can simply use our generic proof protocol to devise more complex protocols.

Some of the changes to the TPM 2.0 interfaces we propose have already been adopted by the TCG and will appear in the forthcoming revision of the TPM 2.0 specifications. The remaining changes are currently under review by the TPM working group. Furthermore, the authors are in discussion with the other bodies standardizing DAA protocols to adopt our changes and schemes, in particular ISO w.r.t. to ISO/IEC 20008-2, Intel for EPID, and with the FIDO alliance for their specification of anonymous attestation [34], so that all of these standards will define provably secure protocols that are compatible with each other.

Outline. We start by presenting the necessary preliminaries in Sec. 2. In Sec. 3, we describe the current TPM 2.0 commands and their inherent security issues and also discuss how previous work aims to overcome these problems. Sec. 4 then presents our proposed changes to the TPM 2.0 specification and our generic proof protocol to create TPM-based attestations. How to build direct anonymous attestation with signature-based revocation and attributes is described in Sec. 5. We discuss forward anonymity separately in Sec. 6, show other applications of the revised TPM interfaces in Sec. 7, and conclude in Sec. 8.

\section{Building BLOCKS AND Assumptions}

This section introduces the notation for signature proofs of knowledge and the complexity assumptions required for our schemes. Here we also present the new generalized version of the LRSW assumption.

\subsection{Bilinear Maps}

Let $\mathbb{G}_{1}, \mathbb{G}_{2}$, and $\mathbb{G}_{T}$ be groups of prime order $p$. A bilinear map $e: \mathbb{G}_{1} \times \mathbb{G}_{2} \rightarrow \mathbb{G}_{T}$ must satisfy bilinearity, i.e., $e\left(g_{1}^{x}, g_{2}^{y}\right)=e\left(g_{1}, g_{2}\right)^{x y}$ for all $x, y \in \mathbb{Z}_{q}$; non-degeneracy, i.e., for all generators $g_{1} \in \mathbb{G}_{1}$ and $g_{2} \in \mathbb{G}_{2}, e\left(g_{1}, g_{2}\right)$ generates $\mathbb{G}_{T}$; and efficiency, i.e., there exists an efficient algorithm $\mathcal{G}\left(1^{\tau}\right)$ that outputs the bilinear group $\left(p, \mathbb{G}_{1}, \mathbb{G}_{2}, \mathbb{G}_{T}, e, g_{1}, g_{2}\right)$ and an efficient algorithm to compute $e(a, b)$ for any $a \in \mathbb{G}_{1}$, $b \in \mathbb{G}_{2}$.

Galbraith et al. [12] distinguish three types of pairings: Type-1, in which $\mathbb{G}_{1}=\mathbb{G}_{2}$; Type-2, in which $\mathbb{G}_{1} \neq \mathbb{G}_{2}$ and there exists an efficient isomorphism $\psi: \mathbb{G}_{2} \rightarrow \mathbb{G}_{1}$; and Type3 , in which $\mathbb{G}_{1} \neq \mathbb{G}_{2}$ and no such isomorphism exists. Type3 pairings currently allow for the most efficient operations in $\mathbb{G}_{1}$ given a security level using Barreto-Naehrig curves with a high embedding degree [13]. Therefore it is desirable to describe a cryptographic scheme in a Type-3 setting, i.e., without assuming $\mathbb{G}_{1}=\mathbb{G}_{2}$ or the existence of an efficient isomorphism from $\mathbb{G}_{2}$ to $\mathbb{G}_{1}$.

\subsection{Complexity Assumptions}

We recall some existing complexity assumptions and introduce a variation of one of them (which we prove to hold in the generic group model). Let $\mathcal{G}\left(1^{\tau}\right)$ generate random groups $\mathbb{G}_{1}=\left\langle g_{1}\right\rangle, \mathbb{G}_{2}=\left\langle g_{2}\right\rangle, \mathbb{G}_{T}=\left\langle e\left(g_{1}, g_{2}\right)\right\rangle$, all of prime order $p$ where $p$ has bith length $\tau$, with bilinear map $e$.

Recall the $q$-SDH assumption [14] and the LRSW assumption [15] in a bilinear group.

Assumption 1 ( $q$-SDH). Define the advantage of $\mathcal{A}$ as:

$$
\begin{aligned}
& \operatorname{Adv}(\mathcal{A})=\operatorname{Pr}\left[\left(\mathbb{G}_{1}, \mathbb{G}_{2}, \mathbb{G}_{T}, e, q\right) \leftarrow \mathcal{G}\left(1^{\tau}\right), x \leftarrow \mathbb{Z}_{p}^{*},\right. \\
& \left.(c, h) \leftarrow \mathcal{A}\left(g_{1}, g_{1}^{x}, g_{1}^{\left(x^{2}\right)}, \ldots, g_{1}^{\left(x^{q}\right)}, g_{2}, g_{2}^{x}\right): h=g_{1}^{\frac{1}{x+c}}\right] .
\end{aligned}
$$

No PPT adversary has $\operatorname{Adv}(\mathcal{A})$ non-negligible in $\tau$.

Assumption 2 (LRSW). Let $X=g_{2}^{x}$ and $Y=g_{2}^{y}$, and let $\mathcal{O}_{X, Y}(\cdot)$ be an oracle that, on input a value $m \in \mathbb{Z}_{p}$, outputs 
a triple $\left(a, a^{y}, a^{x+x y m}\right)$ for a randomly chosen $a$. Define the advantage of $\mathcal{A}$ as follows:

$$
\begin{gathered}
\operatorname{Adv}(\mathcal{A})=\operatorname{Pr}\left[\left(\mathbb{G}_{1}, \mathbb{G}_{2}, \mathbb{G}_{T}, e, q\right) \leftarrow \mathcal{G}\left(1^{\tau}\right),(x, y) \leftarrow \mathbb{Z}_{p}^{2},\right. \\
X \leftarrow g_{2}^{x}, Y \leftarrow g_{2}^{y},(a, b, c, m) \leftarrow \mathcal{A}^{\mathcal{O}_{X, Y}(\cdot)}(X, Y): \\
\left.m \notin Q \wedge a \in \mathbb{G}_{1} \wedge a \neq 1_{\mathbb{G}_{1}} \wedge b=a^{y} \wedge c=a^{x+x y m}\right] .
\end{gathered}
$$

No PPT adversary has $\operatorname{Adv}(\mathcal{A})$ non-negligible in $\tau$.

We introduce a generalized version of the LRSW assumption where we split the oracle $\mathcal{O}_{X, Y}$ into one that first gives the values $a$ and $b$, the two elements that do not depend on the message, and one that later provides $c$ upon input of $m$. That is, after receiving $a, b$, the adversary may specify a message $m$ to receive $c=a^{x+x y m}$.

Assumption 3 (Generalized LRSW). Let $X=g_{2}^{x}$ and $Y=$ $g_{2}^{y}$, and let $\mathcal{O}_{X}^{\mathrm{a}, \mathrm{b}}(\cdot)$ return $(a, b)$ with $a \leftarrow \mathbb{G}_{1}$ and $b \leftarrow a^{y}$. Let $\mathcal{O}_{X, Y}^{\mathrm{c}}(\cdot)$ on input $(a, b, m)$, with $(a, b)$ generated by $\mathcal{O}_{X, Y}^{\mathrm{a}, \mathrm{b}}$, output $c=a^{x+x y m}$. It ignores queries with input $(a, b)$ not generated by $\mathcal{O}_{X, Y}^{\mathrm{a}, \mathrm{b}}$ or inputs $(a, b)$ that were queried before. Define the advantage of $\mathcal{A}$ as follows.

$$
\begin{gathered}
\operatorname{Adv}(\mathcal{A})=\operatorname{Pr}\left[\left(\mathbb{G}_{1}, \mathbb{G}_{2}, \mathbb{G}_{T}, e, q\right) \leftarrow \mathcal{G}\left(1^{\tau}\right),(x, y) \leftarrow \mathbb{Z}_{p}^{2},\right. \\
X \leftarrow g_{2}^{x}, Y \leftarrow g_{2}^{y},(a, b, c, m) \leftarrow \mathcal{A}_{X}^{\mathcal{O}_{X}^{\mathrm{a} b}(\cdot), \mathcal{O}_{X, Y}^{c}(\cdot)}(X, Y): \\
\left.m \notin Q \wedge a \in \mathbb{G}_{1} \wedge a \neq 1_{\mathbb{G}_{1}} \wedge b=a^{y} \wedge c=a^{x+x y m}\right]
\end{gathered}
$$

No PPT adversary has $\operatorname{Adv}(\mathcal{A})$ non-negligible in $\tau$.

Note that our assumption implies the LRSW assumption, but the contrary is not true. In our assumption, the adversary may let $m$ depend on $(a, b)$. Intuitively, it is clear that this does not give any meaningful advantage, as $a$ is random in $\mathbb{G}_{1}$. We formalize this intuition and prove that Assumption 3 holds in Shoup's generic group model [16] in the full version of this paper.

\subsection{Proof Protocols}

For zero-knowledge proofs of knowledge of discrete logarithms and statements about them, we will follow the notation introduced by Camenisch and Stadler [19] and formally defined by Camenisch, Kiayias, and Yung [20]. For instance, $\operatorname{PK}\left\{(a): y=g^{a}\right\}$ denotes a "zero-knowledge Proof of Knowledge of integer a such that $y=g^{a}$ holds." SPK $\{\ldots\}(m)$ denotes a signature proof of knowledge on $m$, that is a noninteractive transformation of a zero-knowledge proof $P K$ with the Fiat-Shamir heuristic [21] in the random oracle model [22].

(S)PK protocols have three moves: In the first move the prover sends to the verifier what is often referred to as a commitment message or $t$-values. In the second move, the verifier sends a random challenge $c$ to which the prover responds with the so-called $s$-values.

When describing our protocols at a high-level, we use the following, more abstract notation. By $\operatorname{NIZK}\{(w)$ : statement $(w)\}(c t x t)$ we denote any non-interactive zeroknowledge proof that is bound to a certain context ctxt and proves knowledge of a witness $w$ such that the statement statement $(w)$ is true.

\section{RELATED WORK \& CURRENT TPM 2.0 SPECIFICATION}

We now summarize the specification of current TPM 2.0 DAA interfaces and discuss its inherent security and privacy issues and how existing work aims to overcome them.

TPM 2.0 Interface and SPKs. For realizing DAA, and signature proofs of knowledge of a TPM secret key in general, the TPM 2.0 specification offers four main commands TPM.Create, TPM.Hash, TPM.Commit, and TPM.Sign. Calling TPM.Create triggers the creation of a secret key $t s k \in \mathbb{Z}_{p}$ and a public key $t p k \leftarrow \bar{g}^{t s k}$, where $\bar{g}$ and $\mathbb{Z}_{p}$ are fixed parameters. Roughly, for signing a message $m$ via a signature proof of knowledge (SPK) of $t s k$ w.r.t. a basename $b s n_{L}$, the host first invokes TPM.Commit on input a group element $g$ and basename $b s n_{L}$ upon which the TPM outputs (commitId, E, K,L) with $K \leftarrow \mathrm{H}_{\mathbb{G}_{1}}\left(b s n_{L}\right)^{t s k}$, and the $t$-values of the SPK, denoted $E \leftarrow g^{r}$ and $L \leftarrow \mathrm{H}_{\mathbb{G}_{1}}\left(b s n_{L}\right)^{r}$. The TPM also internally stores (commitId, $r$ ). The host then calls TPM.Hash to obtain a hash $c$ on the message $(m,(E, L))$. If the TPM wants to sign this message, it marks $c$ as safe to sign. The proof gets completed by invoking the TPM.Sign command on input a safe-to-sign hash $c$ and a reference commitId to the randomness $r$ upon which the TPM outputs $s \leftarrow r+c \cdot t s k$.

Due to this generic interface, the TPM 2.0 can be used to construct multiple DAA schemes. Chen and Li [5] show that the TPM 2.0 supports both LRSW-based DAA [23] and $q$ SDH-based DAA [3], whereas the TPM 1.2 only supported the original RSA-based DAA scheme [1]. Unfortunately, the current TPM 2.0 interfaces have some drawbacks: the signature proofs of knowledge the TPM makes cannot be proven to be unforgeable and there exists a static Diffie-Hellman oracle leaking information about the TPM key.

\subsection{Unforgeability Flaw for TPM 2.0-based SPKs}

The SPKs that are created via the TPM commands should be unforgeable, i.e., a host must not be able to compute an SPK on message $m$ without calling TPM.Sign on a hash $c$ that was previously cleared via a TPM.Hash call on $\mathrm{m}$. Chen and $\mathrm{Li}$ [5] aim to prove this property, but the proof is incorrect, as pointed out by $\mathrm{Xi}$ et al. [8]. In the proof, the authors simulate the TPM without knowing its secret key $t s k$. To simulate an SPK on message $m$, the authors use the standard approach of randomly choosing the $c, s$ values, and then derive the $t$-values $E, L$ in TPM.Commit based on $c, s$, and tpk. For the reduction to go through, one must ensure that the randomly chosen $c$ becomes the hash value of $(m, t)$ (via TPM.Hash and modeling the hash as random oracle), and then let TPM.Sign respond with $s$ whenever that $c$ is given as input. However, given that an adversary has arbitrary access to the TPM interfaces, it can query TPM.Hash on many different messages $\left(m_{1}, t\right), \ldots,\left(m_{n}, t\right)$ containing the same $t$ value. The reduction does not know which of these queries the adversary will later use to complete the signature, and thus only has a $1 / n$ chance to correctly simulate the proof. 
Unforgeability Fix Breaks Privacy. This problem is inherent in the current TPM interface, but could be solved by a simple modification to the TPM.Sign method as proposed by $\mathrm{Xi}$ et al. [8]: when signing, the TPM first chooses a nonce $n_{t}$ and computes $c^{\prime} \leftarrow \mathrm{H}\left(n_{t}, c\right)$ and $s \leftarrow r+c^{\prime} \cdot t s k$. This allows to prove the unforgeability of TPM generated SPKs, as the reduction can now program the random oracle on $c^{\prime}$ only when the TPM.Sign query is made.

However, this would also introduce a subliminal channel for the TPM, as $n_{t}$ would be part of the final signature and a subverted TPM can embed arbitrary information in that nonce, breaking the anonymity without a host noticing. Recent revelations of subverted cryptographic standards and tampered hardware indicate that such attacks are very realistic. We propose changes to the TPM that provably prevent such subliminal challenges and at the same time allow to prove the unforgeability of the SPKs, as we will show in Sec. 4.

\subsection{Static Diffie-Hellman Oracle}

Another problem in the TPM 2.0 interface is the static Diffie-Hellman (DH) oracle, as pointed out by Acar et al. [25]. For any chosen point $g \in \mathbb{G}_{1}$, the host can learn $g^{t s k}$ by calling (commitId, $E, K, L) \leftarrow$ TPM.Commit $(g, b s n), s \leftarrow$ TPM.Sign (commitId, c) and computing $g^{t s k} \leftarrow\left(g^{s} \cdot E^{-1}\right)^{1 / c}$. This leaks a lot of information about $t s k$, Brown and Gallant [26] and Cheon [27] show that the existence of such an oracle makes finding the discrete log much easier. The reason is that the oracle can be used to compute a $q$-SDH sequence $g^{t s k}, g^{t s k^{2}}, \ldots, g^{t s k}$ for very large $q$, which in turn allows to recover $t s k$ faster than had one been given only $\bar{g}^{t s k}$. On Barreto-Naehrig (BN) curves [13], one third of the security strength can be lost due to a static DH oracle. For example, a 256 bit BN curve, which should offer 128 bits of security, only offers 85 bits of security with a static DH oracle.

The static DH oracle also prevents forward anonymity. Forward anonymity guarantees that signatures made by an honest platform remain anonymous, even when the host later becomes corrupted. In existing schemes, even anonymous signatures contain a pair $\left(g_{i}, U_{i, k}\right)$ where $g_{i}$ is a random generator and $U_{i, k}=g_{i}^{t s k_{k}}$. With a static DH oracle, a host upon becoming corrupt can use the TPM to compute $U_{i}^{\prime}=g_{i}^{t s k}$ for all previous signatures, test whether $U_{i}^{\prime}=U_{i, k}$, breaking the anonymity of these signatures.

Cleared Generators for LRSW-based Schemes. Xi et al. [8] propose an approach to remove the static DH oracle while preserving the support for the both LRSW- and $q$-SDH-based DAA schemes. They introduce a new TPM.Bind command that takes as input two group elements $P$ and $K$ and a proof $\pi_{P} \leftarrow$ $\operatorname{SPK}\left\{\alpha: P=\bar{g}^{\alpha} \wedge K=t p k^{\alpha}\right\}$. The TPM verifies the proof and, if correct, stores $P$ as cleared generator. The TPM.Commit interface will then only accept such cleared generators as input for $g$. This removes the static DH oracle because the proof $\pi_{P}$ shows that $P^{t s k}=K$ is already known. A similar approach was also used in the recent LRSW-DAA scheme by Camenisch et al. [9].
However, this approach has two crucial problems. First, it is very hard to implement this functionality on a TPM. The TPM stores only a small number of root keys due to the very limited amount of storage available. For all other keys, the TPM creates a "key blob" that contains the public part of the key in the clear and the private part of the key encrypted with one of the root keys. The TPM would have to similarly store an authenticated list of generators which have been cleared via the TPM.Bind interface. However, this would be a new type of key structure, which is a significant change to the current TPM 2.0 specification.

Second, this interface does not support signature-based revocation, which is an important extension to anonymous signatures. This type of revocation was introduced in EPID [28] and allows one to revoke a platform given a signature from that platform. Roughly, for signature-based revocation, every signature includes a pair $(B$, nym $)$ where $B \leftarrow \mathbb{G}_{1}$ and nym $\leftarrow B^{t s k}$. The signature revocation list SRL contains tuples $\left\{\left(B_{i}, n^{n} m_{i}\right)\right\}$ from signatures of the platforms that are revoked. When signing, the TPM must also prove that it is not the producer of any of these revoked signatures. To do so, it proves $\pi_{\mathrm{SRL}, i} \leftarrow \mathrm{SPK}^{*}\left\{(t s k):\right.$ nym $\left.=B^{\text {tsk }} \wedge n y m_{i} \neq B_{i}^{\text {tsk }}\right\}$ for each tuple in SRL. Using the changes proposed by Xi et al. [8], a host cannot input the generators $B_{i}$ to the TPM anymore as it is not able to produce proofs $\pi_{B_{i}}$ that are required in the TPM.Bind interface.

Random Generators via Hashing. Another approach to remove the static DH oracle is to determine the base $g$ by hashing. That is, instead of inputing $g$ in TPM.Commit, the host provides a basename $b s n_{E}$ upon which the TPM derives $g \leftarrow \mathrm{H}_{\mathbb{G}_{1}}\left(b s n_{E}\right)$. By assuming that the hash function is a random oracle, $g$ is now enforced to be a random instead of a chosen generator which avoids the static $\mathrm{DH}$ oracle, as the host can no longer create the large $q$-SDH sequences that are the basis of the static DH attacks.

Interestingly, this approach was included in the revision from TPM 1.2 to TPM 2.0 to avoid another static DH oracle that was present in the earlier standard. In TPM 1.2, the TPM.Commit interface received a generator $j$ instead of $b s n_{L}$ and directly computed $K \leftarrow j^{\text {tsk }}$ and $L \leftarrow j^{r}$, whereas TPM 2.0 now receives $b s n_{L}$ and first derives $j \leftarrow \mathrm{H}_{\mathbb{G}_{1}}\left(b s n_{L}\right)$.

While applying the same idea on $g$ would solve the problem, it would also significantly limit the functionality of the TPM interface. Recall that TPM 2.0 was designed to support both, LRSW- and $q$-SDH-based DAA schemes. While $q$-SDH schemes could be easily ported to these new interfaces, no current LRSW-based scheme would be supported. All existing LRSW-based schemes require the TPM to prove knowledge of $d=b^{t s k}$ for a generator $b \leftarrow a^{y}$ chosen by the DAA issuer. As the issuer must be privy of the discrete logarithm $y$, it cannot produce a basename $b s n_{E}$ such that $b=\mathrm{H}_{\mathbb{G}_{1}}\left(b s n_{E}\right)$ holds at the same time.

Another protocol that would, in its current forms, not be compatible with this change is the aforementioned signaturebased revocation [28], which needs the TPM to use basenames 
$B_{i}$ defined in the revocation list SRL. Camenisch et al. [10] recently proposed to use $B \leftarrow \mathrm{H}_{\mathbb{G}_{1}}(b s n)$ instead of $B \leftarrow \mathbb{G}_{1}$ to avoid the DH oracle, i.e., the TPM gets $b s n$ as input and the SRL has the form $\left\{\left(b s n_{i}, n y m_{i}\right)\right\}$. However, the authors did not detail how the TPM interfaces have to be changed to support this approach. In fact, their protocol is not easily instantiable, as their proposed computations by the TPM for generating the proofs $\pi_{\mathrm{SRL}, i}$ would require the TPM to keep state, which in turn would require new TPM commands.

Our Approach. In this work we follow the idea of using hash-based generators but thoroughly describe the necessary changes to the TPM 2.0 specification and, in addition, are very conscious to optimize our solutions. Most importantly, our proposed modifications do not require any new TPM commands, but modify the existing ones only slightly. To demonstrate the flexibility of our TPM interface we present a generic protocol that allows to create a wide class of signature proofs of knowledge using these TPM commands. The existing LRSW-based DAA and signature-based revocation protocols cannot be used with our interface due to the aforementioned issues. We therefore also propose new protocols for signaturebased revocation and LRSW-based DAA that are compatible with the proposed TPM interfaces and provably secure.

\section{The Revised TPM 2.0 Interface}

This section introduces new TPM 2.0 interfaces for creating signature proofs of knowledge. The TPM creates keys with the TPM.Create command. Messages can be signed by first calling TPM.Commit, followed by a TPM.Hash and a TPM.Sign command. We first discuss our proposed modifications to these commands and how they address the problems mentioned in Sec. 3. Indeed, we are able to do that by making only minor modifications to the commands. The description of our revised TPM interfaces is presented in Fig. 1. We again use a simplified notation and refer for the full specification of our TPM 2.0 interfaces to the full version of this paper.

Avoiding a Subliminal Channel. To solve the unforgeability problem discussed in Sec. 3, a nonce to which the TPM contributed needs to be included in the computation of the Fiat-Shamir challenge $c^{\prime}$. Thereby, a malicious TPM must not be able to alter the distribution of the signature proofs of knowledge, as this would break the privacy, which is the key goal of anonymous attestation. For this reason, the nonce needs to be computed jointly at random by the TPM and the host. In TPM.Commit, the TPM chooses a nonce $n_{t}$ and commits to that nonce by computing $\bar{n}_{t} \leftarrow \mathrm{H}$ ("nonce", $\left.n_{t}\right)$. The host picks another nonce $n_{h}$, and gives that as input to TPM.Sign. The TPM must use $n_{t} \oplus n_{h}$ in the Fiat-Shamir hash. An honest host takes $n_{h}$ uniformly at random, which guarantees that $n_{t} \oplus n_{h}$ is uniform random, preventing a malicious TPM from hiding messages in the nonce.

Avoiding the DH Oracle. The TPM.Commit command is changed to prevent a static Diffie-Hellman oracle. The oracle exists in the current TPM 2.0 interface because therein a host can pass any value $g$ to the TPM and obtain $g^{t s k}$. Our revised TPM prevents this as it will only use a generator $\tilde{g}$ that is either $\tilde{g} \leftarrow \mathrm{H}_{\mathbb{G}_{1}}\left(b s n_{E}\right)$ for some $b s n_{E}$ it receives, or set to $\tilde{g} \leftarrow \bar{g}$ if $b s n_{E}=\perp$ where $\bar{g}$ denotes the fixed generator used within the TPMs.

Clearly, the host can no longer abuse this interface to learn information about the TPM secret key tsk. If $\tilde{g}=\bar{g}$, the host receives $t p k$ which it already knows. If $\tilde{g}=\mathrm{H}_{\mathbb{G}_{1}}\left(b s n_{E}\right)$ and we model the hash function as a random oracle, the host receives a random element raised to power $t s k$, which does not give the host useful information. More precisely, the proof of Lemma 2 shows that we can simulate a TPM without knowing $t s k$, which proves that the TPM does not leak information on $t s k$. Although our changes limit the generators that the host can choose, Sec. 5.2 shows that we can still build DAA schemes based on $q$-SDH and LRSW on top of this interface, including support for signature-based revocation.

\subsection{Zero-knowledge Proofs with the TPM}

We now describe how our proposed TPM interfaces can be used to create a wide class of signature proofs of knowledge. To demonstrate the flexibility of our interface we propose a generic proof protocol Prove that orchestrates the underlying TPM commands. We then show that proofs generated by Prove are unforgeable, device-bound and remain zero-knowledge even if the TPM is subverted. Thus, protocol designers can use our Prove protocol as generic building block for more complex protocols instead of having to use the TPM command and proving these security properties from scratch. Our DAA protocols presented in Sec. 5 use exactly that approach.

A Generic Prove Protocol. Using the proposed TPM interfaces, a host can create signature proofs of knowledge of the following structure:

$$
\begin{gathered}
\operatorname{SPK}^{*}\left\{\left(\gamma \cdot(t s k+h s k), \alpha_{1}, \ldots, \alpha_{l}\right):\right. \\
y_{1}=\left(\hat{g}^{\delta}\right)^{\gamma \cdot(t s k+h s k)} \cdot \prod_{i} b_{i}^{\alpha_{i}} \wedge \\
y_{2}=\mathrm{H}_{\mathbb{G}_{1}}\left(b s n_{L}\right)^{\gamma \cdot(t s k+h s k)} \cdot \prod_{i} b_{i}^{\prime \alpha_{i}} \wedge \\
\left.y_{3}=\prod_{i} b_{i}^{\prime \prime \alpha_{i}}\right\}\left(m_{h}, m_{t}\right),
\end{gathered}
$$

for values $\delta, h s k, t s k$, and $\gamma$ in $\mathbb{Z}_{p}$, strings $b s n_{L}, m_{h}, m_{t} \in$ $\{0,1\}^{*}$, group elements $y_{1}, y_{2}, y_{3}, \hat{g}$, and set $\left\{\left(\alpha_{i}, b_{i}, b_{i}^{\prime}, b_{i}^{\prime \prime}\right)\right\}_{i}$, with $\alpha_{i} \in \mathbb{Z}_{p}$. Either $y_{1}, \hat{g}$, and all $b_{i}$ 's are in $\mathbb{G}_{1}$ or they are all in $\mathbb{G}_{T}$. All $b_{i}^{\prime}$ values and $y_{2}$ must be in $\mathbb{G}_{1}$. If $b s n_{L}=\perp$, the second equation proving a representation of $y_{2}$ is omitted from the proof. We could also lift this part of the proof to $\mathbb{G}_{T}$ but as we do not require such proofs, we omit this to simplify the presentation. The values $y_{3}$ and $b_{i}^{\prime \prime}$ must either all be in $\mathbb{G}_{1}$, in $\mathbb{G}_{2}$, or in $\mathbb{G}_{T}$.

In addition we require that the TPM works with a cleared generator, meaning either $\hat{g}=\tilde{g}$ or $\hat{g}=e\left(\tilde{g}, \hat{g}_{2}\right)$ with $\tilde{g}$ denoting the cleared generator being either $\bar{g}$, i.e., the fixed generator or it is $\mathrm{H}_{\mathbb{G}_{1}}\left(b s n_{E}\right)$ for some $b s n_{E}$. 
Session system parameters: $\mathbb{G}_{1}=\langle\bar{g}\rangle$ of prime order $q$, nonce bit length $l_{n}$, random oracles $\mathrm{H}:\{0,1\}^{*} \rightarrow \mathbb{Z}_{p}$ and $\mathrm{H}_{\mathbb{G}_{1}}:\{0,1\}^{*} \rightarrow$ $\mathbb{G}_{1}$. Initialize Committed $\leftarrow \emptyset$ and commitId $\leftarrow 0$.

Init. On input TPM.Create():

- If this is the first invocation of TPM.Create, choose a fresh secret key $t s k \leftarrow \mathbb{Z}_{p}$ and compute public key $t p k \leftarrow \bar{g}^{t s k}$.

- Store $t s k$ and output $t p k$.

Hash. On input TPM.Hash $\left(m_{t}, m_{h}\right)$ :

- If $m_{t} \neq \perp$, the TPM checks whether it wants to attest to $m_{t}$.

- Compute $c \leftarrow \mathrm{H}\left(\right.$ "TPM", $\left.m_{t}, m_{h}\right)$.

- Mark $c$ as "safe to sign" and output $c$.
Commit. On input TPM.Commit $\left(b s n_{E}, b s n_{L}\right)$ :

- If $b s n_{E} \neq \perp$, set $\tilde{g} \leftarrow \mathrm{H}_{\mathbb{G}_{1}}\left(b s n_{E}\right)$, otherwise set $\tilde{g} \leftarrow \bar{g}$.

- Choose $r \stackrel{s}{\leftarrow} \mathbb{Z}_{p}, n_{t} \stackrel{s}{\leftarrow}\{0,1\}^{l_{n}}$ and store (commitId, $\left.r, n_{t}\right)$ in Committed.

- Set $\bar{n}_{t} \leftarrow \mathrm{H}$ ("nonce", $\left.n_{t}\right), E \leftarrow \tilde{g}^{r}$, and $K, L \leftarrow \perp$.

- If $b s n_{L} \neq \perp$, set $j \leftarrow \mathrm{H}_{\mathbb{G}_{1}}\left(b s n_{L}\right), K \leftarrow j^{\text {tsk }}$ and $L \leftarrow j^{r}$.

- Output (commitId, $\left.\bar{n}_{t}, E, K, L\right)$ and increment commitId.

Sign. On input TPM.Sign (commitId, $\left.c, n_{h}\right)$ :

- Retrieve record (commitId, $r, n_{t}$ ) and remove it from Committed, output an error if no such record was found.

- If $c$ is safe to sign, set $c^{\prime} \leftarrow \mathrm{H}\left(" F S\right.$ ", $\left.n_{t} \oplus n_{h}, c\right)$ and $s \leftarrow r+c^{\prime} \cdot t s k$ and output $\left(n_{t}, s\right)$.

Fig. 1. Our proposed modified TPM 2.0 interface (changes w.r.t. the current specification are highlighted in blue).

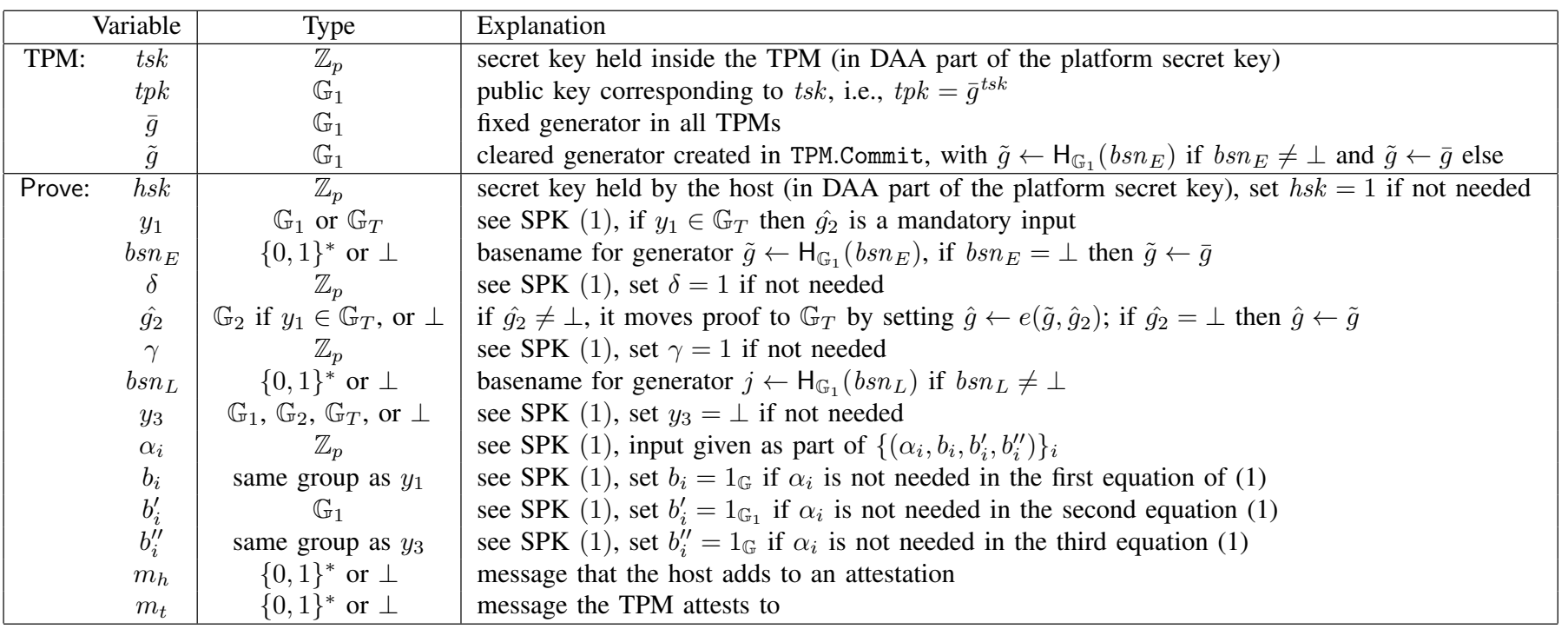

Fig. 2. Overview of variables used within the TPM and in our Prove protocol.

The protocol allows the host to add a key hsk to the witness for $t s k$ because, as we will see in the later sections, this can improve the privacy of DAA schemes. Note that we could trivially generalize the proof statement (1) to include additional terms that do not contain $\gamma \cdot(t s k+h s k)$ as witness, but for ease of presentation we omit these additional terms.

The host can add any message $m_{h}$ to the proof. It also chooses $m_{t}$, but this is a value the TPM attests to and will be checked by the TPM.

The host can create such a proof using the Prove protocol described in Fig. 3. We assume a perfectly secure channel between the host and TPM, i.e., the adversary does not notice the host calling TPM commands. Note that before starting the proof, the host may not know $y_{2}$, as it does not know tsk, but learns this value during the proof because it is given as output of the Prove protocol. How to verify such proofs using the VerSPK algorithm is shown in Fig. 3 as well. Note that verification does not require any participation of the TPM. Fig. 2 gives a brief overview of the required parameters and their respective types and conditions.

The completeness of these proofs can easily be verified.
The proof is sound as we can extract a valid witness using the standard rewinding technique.

Example for Using Prove. We now give a simple example to show how the Prove protocol must be invoked and give some intuition on how the final proof is assembled by our protocol. Suppose we want to prove:

$$
\begin{aligned}
\mathrm{SPK}^{*}\left\{(t s k+h s k): d^{\prime}\right. & =\left(\mathrm{H}_{\mathbb{G}_{1}}\left(b s n_{E}\right)^{\delta}\right)^{(t s k+h s k)} \wedge \\
n y m & \left.=\mathrm{H}_{\mathbb{G}_{1}}\left(b s n_{L}\right)^{(t s k+h s k)}\right\}\left(m_{h}, m_{t}\right),
\end{aligned}
$$

where the TPM holds tsk and the host knows $h s k$. The host will add $h s k$ to the witness for $t s k$, which is the first input to Prove. The second argument is the left hand side of the first equation, which is $d^{\prime}$. The generator for the witness $t s k+h s k$ is $\left(\mathrm{H}_{\mathbb{G}_{1}}\left(b s n_{E}\right)^{\delta}\right)$, which is passed on to the Prove protocol by giving $b s n_{E}$ and $\delta$ as the next arguments. The protocol has the option to move the proof to $\mathbb{G}_{T}$ by passing a value $\hat{g}_{2}$, but as this proof takes place in $\mathbb{G}_{1}$, we enter $\hat{g}_{2}=\perp$. We can prove knowledge of $\gamma \cdot(t s k+h s k)$, but as we want to use witness $t s k+h s k$, we pass $\gamma=1$. In the second equation, we use $\mathrm{H}_{\mathbb{G}_{1}}\left(b s n_{L}\right)$ as generator, so we give argument $b s n_{L}$. 
Prove $\left(h s k, y_{1}, b s n_{E}, \delta, \hat{g}_{2}, \gamma, b s n_{L}, y_{3}\right.$,

$\left.\left\{\left(\alpha_{i}, b_{i}, b_{i}^{\prime}, b_{i}^{\prime \prime}\right)\right\}_{i}, m_{h}, m_{t}\right):$

- If $b s n_{E} \neq \perp$, set $\tilde{g} \leftarrow \mathrm{H}_{\mathbb{G}_{1}}\left(b s n_{E}\right)$, otherwise set $\tilde{g} \leftarrow \bar{g}$.

- If $\hat{g}_{2} \neq \perp$, set $\hat{g} \leftarrow e\left(\tilde{g}, \hat{g}_{2}\right)$, otherwise set $\hat{g} \leftarrow \tilde{g}$.

- If $b s n_{L} \neq \perp$, set $j \leftarrow \mathrm{H}_{\mathbb{G}_{1}}\left(b s n_{L}\right)$.

- Call TPM.Commit $\left(b s n_{E}, b s n_{L}\right) \rightarrow\left(\right.$ commitId, $\left.\bar{n}_{t}, E, K, L\right)$.

- Take $r_{h s k} \leftarrow \mathbb{Z}_{p}$, set $E^{\prime} \leftarrow\left(E \cdot \tilde{g}^{r_{h s k}}\right)^{\gamma \cdot \delta}$. If $b s n_{L} \neq \perp$, set $K^{\prime} \leftarrow\left(K \cdot j^{h s k}\right)^{\gamma}$ and $L^{\prime} \leftarrow\left(L \cdot j^{r_{h s k}}\right)^{\gamma}$.

- If $b s n_{L} \neq \perp$, set $y_{2} \leftarrow K^{\prime} \cdot \prod_{i} b_{i}^{\prime \alpha_{i}}$.

- Take $\left\{r_{\alpha_{i}}\right\}_{i=1}^{l} \leftarrow \mathbb{Z}_{p}^{l}$. Set $t_{1} \leftarrow E^{\prime} \cdot \prod_{i} b_{i}^{r_{\alpha_{i}}}$ if $b_{i} \in \mathbb{G}_{1}$, or $t_{1} \leftarrow e\left(E^{\prime}, \hat{g}_{2}\right) \cdot \prod_{i} b_{i}^{r_{\alpha_{i}}}$ if $b_{i} \in \mathbb{G}_{T}$.

- If $b s n_{L} \neq \perp$, set $t_{2} \leftarrow L^{\prime} \prod_{i} b_{i}^{\prime r_{\alpha_{i}}}$ and $t_{2} \leftarrow \perp$ else.

- If $y_{3} \neq \perp$, set $t_{3} \leftarrow \prod_{i} b_{i}^{\prime \prime \prime} r_{\alpha_{i}}$ and $t_{3} \leftarrow \perp$ else.

- Set $m_{h}^{\prime} \leftarrow\left(m_{h}, y_{1}, \hat{g}^{\delta},\left\{\left(b_{i}, b_{i}^{\prime}, b_{i}^{\prime \prime}\right)\right\}, t_{1}, y_{2}, b s n_{L}, t_{2}, y_{3}, t_{3}\right)$.

- Call TPM.Hash $\left(m_{t}, m_{h}^{\prime}\right) \rightarrow c$.

- Take $n_{h} \leftarrow$ $\{0,1\}^{l_{n}}$.

- Call TPM.Sign $\left(\right.$ commitId $\left., c, n_{h}\right) \rightarrow\left(n_{t}, s\right)$.

- Check that $\bar{n}_{t}=\mathrm{H}\left(\right.$ "nonce", $\left.n_{t}\right)$ and set $n \leftarrow n_{h} \oplus n_{t}$, $c^{\prime} \leftarrow \mathrm{H}(" F S$ ", $n, c)$.

- Set $s^{\prime} \leftarrow \gamma \cdot\left(s+r_{h s k}+c^{\prime} \cdot h s k\right)$ and $s_{\alpha_{i}} \leftarrow r_{\alpha_{i}}+c^{\prime} \cdot \alpha_{i}$ for $i=1, \ldots, l$.

- Check $\left(\hat{g}^{\delta}\right)^{s^{\prime}}=E^{\prime} \cdot\left(y_{1} /\left(\prod_{i} b_{i}^{\alpha_{i}}\right)^{c^{\prime}}\right.$ and if $b s n_{E} \neq \perp$, check $j^{s^{\prime}}=L^{\prime} \cdot K^{\prime c^{\prime}}$.

- Set proof $\pi \leftarrow\left(c^{\prime}, n, s^{\prime},\left\{s_{\alpha_{i}}\right\}\right)$ and output $\left(y_{2}, \pi\right)$.

$\operatorname{VerSPK}\left(\pi, y_{1}, \hat{g}^{\delta}, y_{2}, b s n_{L}, y_{3},\left\{\left(\alpha_{i}, b_{i}, b_{i}^{\prime}, b_{i}^{\prime \prime}\right)\right\}_{i}, m_{h}, m_{t}\right):$

- Parse $\pi$ as $\left(c^{\prime}, n, s^{\prime},\left\{s_{\alpha_{i}}\right\}\right)$.

- Set $t_{1} \leftarrow y_{1}^{-c^{\prime}} \cdot\left(\hat{g}^{\delta}\right)^{s^{\prime}} \cdot \prod_{i} b_{i}{ }^{s_{\alpha_{i}}}$.

- If $b s n_{L} \neq \perp$, set $t_{2} \leftarrow y_{2}^{-c^{\prime}} \cdot \mathrm{H}_{\mathbb{G}_{1}}\left(b s n_{L}\right)^{s^{\prime}} \cdot \prod_{i} b_{i}^{\prime s_{\alpha_{i}}}$, and $t_{2} \leftarrow \perp$ else.

- If $y_{3} \neq \perp$, set $t_{3} \leftarrow y_{3}^{-c^{\prime}} \cdot \prod_{i} b_{i}^{\prime \prime s_{\alpha_{i}}}$ and $t_{3} \leftarrow \perp$ else.

- Output 1 if $c^{\prime}=\mathrm{H}$ ("FS", $n, \mathrm{H}$ ("TPM", $m_{t},\left(m_{h}, y_{1}, \hat{g}^{\delta}\right.$, $\left.\left.\left.\left\{\left(b_{i}, b_{i}^{\prime}, b_{i}^{\prime \prime}\right)\right\}, t_{1}, y_{2}, b s n_{L}, t_{2}, y_{3}, t_{3}\right)\right)\right)$, and 0 otherwise.

Fig. 3. Prove protocol and VerSPK algorithm to create and verify zeroknowledge proofs via the TPM interfaces from Fig. 1.

Since our proof omits the third equation, we set $y_{3} \leftarrow \perp$. The protocol supports an additional list of witnesses with generators in the three equations, but since this equation only uses witness $t s k+h s k$, we pass an empty list as next argument. Finally, we specify $m_{t}$, the message the TPM attests to, and $m_{h}$, the additional data added by the host. Therefore, we call

$$
\text { Prove }\left(h s k, d^{\prime}, b s n_{E}, \delta, \perp, 1, b s n_{L}, \perp, \emptyset, m_{h}, m_{t}\right) .
$$

The protocol calls TPM.Commit with basenames $b s n_{E}$ and $b s n_{L}$ to receive $E=\mathrm{H}_{\mathbb{G}_{1}}\left(b s n_{E}\right)^{r_{t s k}}$ and $L=\mathrm{H}_{\mathbb{G}_{1}}\left(b s n_{L}\right)^{r_{t s k}}$ for some $r_{t s k}$, and $K=\mathrm{H}_{\mathbb{G}_{1}}\left(b s n_{L}\right)^{t s k}$, along with $\bar{n}_{t}=$ $\mathrm{H}$ ("nonce", $\left.n_{t}\right)$, that commits the TPM to TPM nonce $n_{t}$. The host must change the generator for the first proof equation to $\mathrm{H}_{\mathbb{G}_{1}}\left(b s n_{L}\right)^{\delta}$ instead of $\mathrm{H}_{\mathbb{G}_{1}}\left(b s n_{L}\right)$, and add randomness to both values to prevent a malicious TPM from altering the distribution of the resulting proof. It sets $t_{1} \leftarrow E^{\delta}$. $\left(\mathrm{H}_{\mathbb{G}_{1}}\left(b s n_{E}\right)^{\delta}\right)^{r_{s s k}}=\left(\mathrm{H}_{\mathbb{G}_{1}}\left(b s n_{E}\right)^{\delta}\right)^{r_{t s k}+r_{t s k}}$, and $t_{2} \leftarrow L$. $\mathrm{H}_{\mathbb{G}_{1}}\left(b s n_{L}\right)^{r_{h s k}}=\mathrm{H}_{\mathbb{G}_{1}}\left(b s n_{E}\right)^{r_{t s k}+r_{t s k}}$. Next, it hashes the $t$ values along with the proof parameters and messages $m_{t}$ and $m_{h}$ using TPM.Hash. The TPM inspects $m_{t}$ and returns $c$, which can only be passed to TPM.Sign if the TPM agrees to signing $m_{t}$. The host now calls TPM.Sign with $c$ and a fresh host nonce $n_{h}$, upon which it receives $n_{t}$ and $s=r_{t s k}+c^{\prime} \cdot t s k$. The host checks whether $n_{t}$ matches the committed TPM nonce, and computes the joint nonce $n \leftarrow n_{h} \oplus n_{t}$ and FiatShamir challenge $c^{\prime} \leftarrow \mathrm{H}(" F S$ ", $n, c)$. The host must now add its randomness and $h s k$ to the $s$-value, which it does by setting $s^{\prime} \leftarrow s+r_{h s k}+c^{\prime} \cdot h s k$. Finally, it checks whether the resulting proof is valid, to make sure that the TPM contributions did not invalidate the proof. The resulting proof consists of nonce $n$, Fiat-Shamir challenge $c^{\prime}$, and $s$-value $s^{\prime}$.

\subsubsection{Security of Prove}

We now show that proofs generated by our generic Prove protocol specified in Fig. 3 and using the TPM interfaces as described in Fig. 1 are unforgeable, device-bound and remain zero-knowledge even if the TPM is subverted.

Zero-knowledge of SPKs with a Corrupt TPM. An SPK created with the Prove protocol is zero knowledge in the random oracle model, even when the TPM is corrupt. That is, we prove the absence of any subliminal channel that a malicious TPM could use to break the privacy of the platform. In Sec. 5 we show that this allows one to devise DAA schemes that guarantee privacy even when the TPM is malicious.

Lemma 1 (Privacy of SPKs with a TPM). The signature proofs of knowledge generated by Prove as defined in Fig. 1, are zero-knowledge, even when the TPM is corrupt.

Proof (sketch). A corrupt TPM may block the creation of the proof, but if it succeeds, it is zero knowledge. The TPM is involved in proving knowledge of $\gamma \cdot(t s k+h s k)$. The host changes the $r$-value to $\gamma \cdot\left(r_{t s k}+r_{h s k}\right)$, with $r_{h s k}$ chosen by the host. It takes $r_{h s k} \leftarrow \mathbb{Z}_{p}$, so $r_{t s k}+r_{h s k}$ is uniform in $\mathbb{Z}_{p}$ regardless of how the TPM chooses $r_{t s k}$. Since $\gamma \neq 0$, $\gamma \cdot\left(r_{t s k}+r_{h s k}\right)$ is still uniform in $\mathbb{Z}_{p}$.

The TPM also chooses a nonce $n_{t}$. It must first commit to this nonce with $\bar{n}_{t}=\mathrm{H}$ ("nonce", $n_{t}$ ). The host then chooses a nonce $n_{h}$ uniformly at random in $\{0,1\}^{l_{n}}$, and the TPM must work with $n=n_{h} \oplus n_{t}$, and show that it computed this correctly. Clearly, $n$ is uniform if $n_{h}$ is uniform.

Since we know the distribution of every part of the zeroknowledge proof, even when the TPM is corrupt, we can simulate proofs of an honest host with a corrupt TPM.

Unforgeability of SPKs with an Honest TPM. We now show that proofs generated by Prove are unforgeable with respect to $m_{t}$, i.e., if the TPM is honest, a corrupt host cannot create a SPK for message $m_{t}$ that the TPM did not approve to sign.

We consider a corrupt host with oracle access to an honest TPM. The TPM executes TPM.Create, outputting $t p k \leftarrow \bar{g}^{t s k}$. The corrupt host cannot create SPKs of structure (1) where $t s k$ is protected by the TPM and $\gamma$ and $h s k$ are known and the TPM never signed $m_{t}$. We require the host to output $\gamma$ and $h s k$ along with his forgery. In a protocol, this means that these 
values must be fixed (e.g., $\gamma$ always equals 1) or extractable from some proof of knowledge for this lemma to be applicable.

Lemma 2 (Unforgeability of SPKs with a TPM). The signature proofs of knowledge generated by Prove as defined in Fig. 1, are unforgeable w.r.t. $m_{t}$. More precisely, the host cannot forge a signature proof of knowledge with the structure of (1) with a witness $\gamma \cdot($ tsk + hsk $)$ for known $\gamma$, hsk if the TPM never signed $m_{t}$, under the DL assumption in the random oracle model.

Proof (sketch). We show that if an adversary $\mathcal{A}$ that has access to the TPM interfaces can forge SPK's, we can derive an adversary $\mathcal{B}$ that can solve the discrete logarithm problem. Note that it is crucial that we allow the adversary $\mathcal{A}$ to get full, unconstrained access to the TPM interfaces instead of giving him only indirect access via the Prove protocol, as this correctly models the power a corrupt host will have.

Our reduction $\mathcal{B}$ receives a DL instance $t p k=\bar{g}^{t s k}$ and is challenged to find $t s k$. To do so, we simulate the TPM and the hash function towards $\mathcal{A}$ based on $t p k, \bar{g}$ as follows:

Hash queries: For queries $b s n_{i}$ to $\mathrm{H}_{\mathbb{G}_{1}}$, take $r_{i} \stackrel{s}{\leftarrow} \mathbb{Z}_{p}$ and return $\mathrm{H}_{\mathbb{G}_{1}}\left(b s n_{i}\right)=\bar{g}^{r_{i}}$ and store (hash, $\left.\mathrm{H}_{\mathbb{G}_{1}}\left(b s n_{i}\right), r_{i}\right)$. Queries to $\mathrm{H}$ and TPM.Hash are handled normally.

Commit query TPM.Commit $\left(b s n_{E}, b s n_{L}\right)$ : Take $\left(s_{i}, c_{i}^{\prime}\right) \stackrel{\$}{\$}$ $\mathbb{Z}_{p}^{2}$. If $b s n_{E} \neq \perp$, compute $\mathrm{H}_{\mathbb{G}_{1}}\left(b s n_{E}\right)$, look up the record (hash, $\left.\mathrm{H}_{\mathbb{G}_{1}}\left(b s n_{E}\right), r_{E}\right)$, and set $E \leftarrow \bar{g}^{s}$. $t p k^{-c_{i}^{\prime} \cdot r_{E}}$. If $b s n_{E}=\perp$, set $E \leftarrow \bar{g}^{s} \cdot t p k^{-c_{i}^{\prime}}$.

If $b s n_{L} \neq \perp$, compute $\mathrm{H}_{\mathbb{G}_{1}}\left(b s n_{L}\right)$, look up the record (hash, $\left.\mathrm{H}_{\mathbb{G}_{1}}\left(b s n_{L}\right), r_{L}\right)$, and set $K \leftarrow t p k^{r_{L}}=$ $\mathrm{H}_{\mathbb{G}_{1}}\left(b s n_{L}\right)^{t s k}$, and $L \leftarrow \bar{g}^{s} \cdot t p k^{-c_{i}^{\prime} \cdot r_{L}}$. If $b s n_{L}=\perp$, set $K \leftarrow \perp$ and $L \leftarrow \perp$.

Pick $\bar{n}_{t}$ uniform in the range of $\mathrm{H}$, store (commitId, $\bar{n}_{t}, s_{i}, c_{i}^{\prime}$ ), increment commitId, and output (commitId, $\left.\bar{n}_{t}, E, K, L\right)$.

Sign query TPM.Sign (commitId, $\left.c, n_{h}\right)$ : Look up and remove record (commitId, $\left.\bar{n}_{t}, s_{i}, c_{i}^{\prime}\right)$, and output an error if no such record was found. Check that $c$ was marked safe-to-sign in a TPM.Hash query. Pick $n_{t} \leftarrow\{0,1\}^{l_{n}}$ and program the random oracle such that $\mathrm{H}\left(\right.$ "nonce", $\left.n_{t}\right)=$ $\bar{n}_{t}$. Program the random oracle such that $\mathrm{H}\left(\right.$ " $F S$ ", $n_{t} \oplus$ $\left.n_{h}, c\right)=c_{i}^{\prime}$. Since the nonce $n_{t}$ is fresh and gets only used once, the probability that the random oracle is already defined on that input is negligible. Finally, we output $\left(n_{t}, s_{i}\right)$.

When $\mathcal{A}$, after having interacted with these oracles, outputs a SPK forgery, i.e., a valid proof with TPM message $m_{t}$ that the TPM never agreed to sign in TPM.Hash, along with values $\gamma, h s k$ such that the proof uses $\gamma \cdot(t s k+h s k)$ as witness, we either have a collision in $\mathrm{H}$ which occurs with negligible probability, or we can rewind to extract $\gamma \cdot(t s k+h s k)$, allowing us to compute $t s k$. $\mathcal{B}$ then outputs $t s k$, solving the DL problem.

Device Boundedness of SPKs with an Honest TPM. Finally, we show that proofs generated via Prove are device bound, i.e., the host cannot create more SPKs than the amount of sign queries the TPM answered. Again, the TPM holds tsk with $t p k=\bar{g}^{t s k}$ created by TPM.Create.

Lemma 3 (Device Boundedness of SPKs with a TPM). The signature proofs of knowledge generated by Prove as defined in Fig. 1, are device bound. That is, the host cannot create more signature proofs of knowledge with the structure of (1) with a witnesses $\gamma \cdot(t s k+h s k)$, where tsk is protected by the TPM and the host knows $\gamma$ and hsk, than the amount of sign queries the TPM answered, under the DL assumption in the random oracle model.

Proof (sketch). Our reduction receives a DL instance tpk $=$ $\bar{g}^{t s k}$ and must compute $t s k$. The simulation works exactly as in the proof of Lemma 2 . If the host made $n$ sign queries and outputs $n+1$ SPKs and corresponding values $\gamma$ and $h s k$, we look at every $c^{\prime}$ value of the proofs. If there are two distinct SPKs with the same $c^{\prime}$ value, there must be a collision in $\mathrm{H}$, which occurs with negligible probability. If all $c^{\prime}$ values are distinct, one of them must be different from the $c^{\prime}$ values as created by the TPM. That means the random oracle is not programmed here and we can rewind that proof to extract $\gamma \cdot(t s k+h s k)$. Since we also have $h s k$ and $\gamma$ we can compute tsk, which solves the DL problem.

\subsubsection{Proofs Without TPM Contribution}

To be able to prove security of our DAA schemes, we must distinguish proofs to which the TPM contributed and proofs that the host created by itself. One way to achieve this is by using different prefixes in the Fiat-Shamir hash computation. Proofs with TPM contribution have a Fiat-Shamir hash $c^{\prime} \leftarrow$ $\mathrm{H}\left(\right.$ "FS", $n, \mathrm{H}\left(\right.$ "TPM", $\left.\left.m_{t}, m_{h}\right)\right)$. Proofs without TPM contribution will use $c^{\prime} \leftarrow \mathrm{H}\left(" F S ", n, \mathrm{H}\left(\right.\right.$ "NoTPM", $\left.\left.m_{t}, m_{h}\right)\right)$. We denote TPM contributed proofs by SPK*, and proofs without TPM contribution SPK.

\section{Provably Secure DAA Schemes}

We now show how to use the proposed TPM interfaces to build provably secure direct anonymous attestation protocols. We start by describing the desired functional and security properties (Sec. 5.1) and then present two DAA protocols, based on the $q-\mathrm{SDH}$ assumption and the LRSW assumption (Sec. 5.2), and argue their security (Sec. 5.3). We refer to Appendix A for the formal definition of DAA in the form of an ideal functionality and to the full version of this paper for the detailed security proof.

\subsection{Definition \& Security Model}

In a DAA scheme, we have four main entities: a number of TPMs, a number of hosts, an issuer, and a number of verifiers. The scheme comprises a JOIN and SIGN protocol, and VERIFY and LINK algorithms.

JOIN: A TPM and a host together form a platform which performs the join protocol with the issuer who decides if the platform is allowed to become a member. The membership credential of the platform then also certifies a number of attributes attrs $=\left(a_{1}, \ldots, a_{L}\right)$ given by the issuer. These 
attributes might include more information about the platform, such as the vendor or model, or other information, such as an expiration date of the credential.

SIGN: Once being a member, the TPM and host together can sign messages $m$ with respect to basename bsn resulting in a signature $\sigma$. If a platform signs with a fresh basename, the signature must be anonymous and unlinkable to previous signatures. When signing, the platform can also selectively disclose attributes from its membership credential. For instance, reveal that the signature was created by a TPM of a certain manufacturer, or the expiration date of the credential. We describe the disclosure using a tuple $(D, I)$ where $D \subseteq\{1, \ldots, L\}$ indicates which attributes are disclosed, and $I=\left(a_{1}, \ldots, a_{L}\right)$ specifies the desired attribute values.

VERIFY: Any verifier can check that a signature $\sigma$ on message $m$ stems from a legitimate platform via a deterministic verify algorithm. More precisely, verification gets as input a tuple $(m, b s n, \sigma,(D, I), \mathrm{RL}, \mathrm{SRL})$ and outputs 1 if $\sigma$ is a valid signatures on message $m$ w.r.t. basename bsn and stems from a platform that has a membership credential satisfying the predicate defined via $(D, I)$, and 0 otherwise.

The inputs RL and SRL are revocation lists and we support two types of revocation, private-key-based revocation and signature-based revocation. The first is based on the exposure of a corrupt platform's secret key (or private key) and allows one to recognize and thus reject all signatures generated with this key. That is, the revocation list RL contains the secret keys of the revoked TPMs. The second type, signature-based revocation, has been proposed by Brickell and Li [28], [29] in their Enhanced Privacy ID (EPID) protocol. It allows one to revoke a platform based on a previous signature from that platform, i.e., here the revocation list SRL contains the signatures of the revoked TPMs.

LINK: By default, signatures created by an DAA scheme do not leak any information about the identity of the signer. Only when the platform signs repeatedly with the same basename $b s n$, it will be clear that the resulting signatures were created by the same platform, which can be publicly tested via the deterministic LINK algorithm. More precisely, on input two signatures $(\sigma, m,(D, I), \mathrm{SRL}),\left(\sigma^{\prime}, m^{\prime},\left(D^{\prime}, I^{\prime}\right), \mathrm{SRL}{ }^{\prime}\right)$, and a basename $b s n$, the algorithm outputs 1 if both signatures are valid and were created by the same platform, and 0 otherwise.

We now describe the desired security properties of DAA schemes in an informal manner. The detailed definition in form of an ideal functionality in the Universal Composability framework [30] is given in Appendix A, and closely follows the recent formal models of Camenisch et al. [9], [24].

Unforgeability. The adversary can only sign in the name of corrupt TPMs. More precisely, if $n$ corrupt and unrevoked TPMs joined with attributes fulfilling attribute disclosure $(D, I)$, the adversary can create at most $n$ unlinkable signatures for the same basename bsn and attribute disclosure $(D, I)$. In particular, this means that when the issuer and all unrevoked TPMs are honest, no adversary can create a valid signature on a message $m$ w.r.t. basename $b s n$ and attribute disclosure $(D, I)$ when no platform that joined with those attributes signed $m$ w.r.t. $b s n$ and $(D, I)$.

Non-Frameability. No adversary can create a signature on a message $m$ w.r.t. basename $b s n$ that links to a signature created by an honest platform, when this honest platform never signed $m$ w.r.t. bsn. We require this property to hold even when the issuer is corrupt.

(Strong) Privacy. An adversary that is given two signatures $\sigma_{1}$ and $\sigma_{2}$ w.r.t. two different basenames $b s n_{1} \neq b s n_{2}$, respectively, cannot distinguish whether both signatures were created by one honest platform, or whether two different honest platforms created the signatures. This property must also hold when the issuer is corrupt.

So far, privacy was conditioned on the honesty of the entire platform, i.e., both the TPM and the host have to be honest. In fact, the previous DAA schemes crucially rely on the honesty of the TPM, and the newly revised TPM interfaces even introduced a subliminal channel that allows a malicious TPM to always encode some identifying information into his signature contribution (see Sec. 3.1). The latter forestalls any privacy in the presence of a corrupt TPM, even if the DAA protocol built on top of the TPM interfaces would allow for better privacy.

In this work we have proposed TPM interfaces that avoid such subliminal channels and we consequently aim for stronger privacy guarantees for DAA as well. That is, the aforementioned indistinguishability of two signatures $\sigma_{1}$ and $\sigma_{2}$ must hold whenever the host is honest, regardless of the corruption state of the TPM. Our notion of strong privacy lies between the classical privacy notion (relying also on the honesty of the TPM) and optimal privacy that was recently introduced by Camenisch et al. [24]. We discuss the differences between these notions, and to [24] in particular, in Appendix A.

\subsection{DAA Protocols}

We start by presenting the high-level idea of both DAA protocols using our revised TPM 2.0 interfaces, and then describe the concrete instantiations based on the $q$-SDH and the LRSW assumption.

Both protocols roughly follow the common structure of previous DAA protocols: the platform, consisting of a TPM and a host, generates a secret key $g s k$ that gets blindly certified by a trusted issuer in a membership credential cred. When attributes attrs $=a_{1}, \ldots a_{L}$ are used, the credential also certifies attrs. After that join procedure, the platform can use the key $g s k$ to sign attestations and basenames and prove that it has a valid credential on the underlying key, which certifies the trusted origin of the attestation. The overview of the DAA protocol is depicted in Fig. 4. 


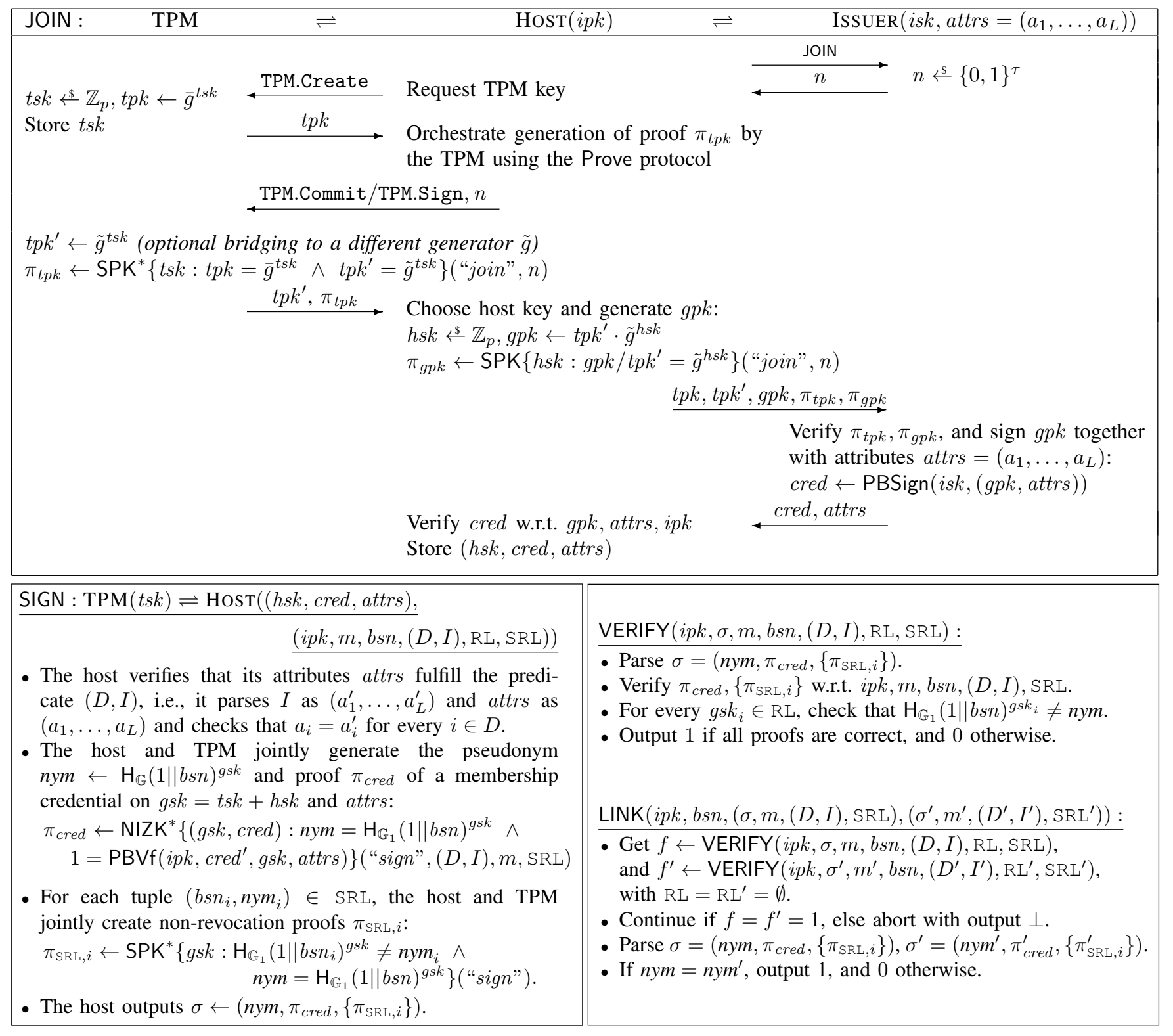

Fig. 4. High-level overview of the DAA protocols.

Split-Keys for Strong Privacy. In contrast to existing schemes, we do not set $g s k=t s k$ because solely relying on the secret key tsk of the TPM would not allow for the strong privacy property we are aiming for. Instead, we partially follow the approach of Camenisch et al. [24] and let the host contribute to the platform's secret key. That is, we split the key as $g s k=t s k+h s k$, where $h s k$ is the contribution of the host to the platform secret key. As in previous work, the platform secret key gsk gets blindly signed by the issuer using a partially blind signature PBSign that certifies the secret key by signing the platform's public key $g p k=\tilde{g}^{g s k}$.

Note that to allow for algorithmic agility, we derive the platform's key from a generator $\tilde{g}$, which can either be a cleared generator created with TPM.Commit as $\tilde{g} \leftarrow \mathrm{H}_{\mathbb{G}_{1}}(0 \| s t r)$ for some string str, or $\tilde{g} \leftarrow \bar{g}$, i.e. being the standard generator fixed in all TPMs. When using a cleared generator, the input to the hash function will be prepended with a 0-bit to ensure that the same generator will not be used in a signature (where we will prepend a 1-bit when creating generators), as this would break the unlinkability between joining and signing otherwise.

We now have to ensure that $g s k$ is derived from a key $t s k$ held inside a real TPM. To this end, the TPM first has to prove in $\pi_{t p k}$ that its contribution $t p k^{\prime}=\tilde{g}^{t s k}$ is based on the same secret key $t s k$ as the actual TPM public key $t p k=\bar{g}^{t s k}$. The host then forwards $t p k, t p k^{\prime}$ and $\pi_{t p k}$ along with a proof $\pi_{g p k}$ that it correctly derived $g p k$ from the TPM's contribution $t p k^{\prime}$ to the issuer.

Each TPM is equipped by the manufacturer with an endorsement key. This key allows the issuer to verify the authenticity of the TPM provided values in the JOIN protocol. As this 
is the standard procedure in all DAA protocols, we omit the details how this authentication is done and implicitly assume that the value tpk in the JOIN protocol is authenticated with the endorsement key.

After having obtained a membership credential on the joint secret key gsk (and possibly a set of attributes attrs), the attestation signatures are then computed jointly by the host and TPM.

Signature-Based Revocation. We also want to support signature-based revocation introduced in the EPID protocol by Brickell and $\mathrm{Li}$ [28], [29] as it allows one to revoke TPMs without assuming that a secret key held inside the TPM becomes publicly available upon corruption, which improves the standard private-key-based revocation in DAA.

Roughly, for signature-based revocation, a platform would extend its signatures by additional values $(B, n y m)$ where $B$ is a random generator for $\mathbb{G}_{1}$ and nym $\leftarrow B^{g s k}$. The signature revocation list SRL contains tuples $\left\{\left(B_{i}, n y m_{i}\right)\right\}$ from signatures of the platforms that are revoked. Thus, a platform must also show that it is not among that list by proving $\pi_{S R L, i} \leftarrow \operatorname{SPK}^{*}\left\{(g s k): n y m=B^{g s k} \wedge n^{\prime} m_{i} \neq B_{i}^{g s k}\right\}$. Any TPM interface that supports such proofs would raise $B_{i}$ to the secret key and inevitably provide a static DH oracle.

Camenisch et al. [10] recently addressed this issue and proposed a $q$-SDH-based DAA scheme with signature-based revocation that avoids this issue. Instead of giving the generator as direct input, it uses $B_{i} \leftarrow \mathrm{H}_{\mathbb{G}_{1}}\left(1 \| b s n_{i}\right)$ computed by the TPM, i.e., the TPM gets $1 \| b s n_{i}$ as input and the SRL has the form $\left\{\left(1 \| b s n_{i}, n_{y m_{i}}\right)\right\}$. For every $\left(1 \| b s n_{i}, n_{y m_{i}}\right) \in \mathrm{SRL}$, the platform shows that $\mathrm{H}_{\mathbb{G}_{1}}\left(1|| b s n_{i}\right)^{g s k} \neq n y m_{i}$ by taking a random $\gamma$, setting $C_{i}=\left(\mathrm{H}_{\mathbb{G}_{1}}\left(1|| b s n_{i}\right) / \text { nym }_{i}\right)^{\gamma}$, and proving

$$
\begin{aligned}
\pi_{\mathrm{SRL}, i}^{\prime} \leftarrow \mathrm{SPK}^{*}\{(\gamma \cdot g s k, \gamma): \\
1=\mathrm{H}_{\mathbb{G}_{1}}(1|| b s n)^{\gamma \cdot g s k}\left(\frac{1}{n y m}\right)^{\gamma} \wedge \\
\left.C_{i}=\mathrm{H}_{\mathbb{G}_{1}}\left(1|| b s n_{i}\right)^{\gamma \cdot g s k}\left(\frac{1}{\text { nym }_{i}}\right)^{\gamma}\right\} \text { ("sign"). }
\end{aligned}
$$

While the proposed scheme successfully removes the static DH oracle and is provably secure in the UC model, their protocol makes different calls to the TPM to prove non-revocation, and requires the TPM to maintain state (bsn,nym) that it used in the signing procedure to later create the non-revocation proofs. Extra TPM commands would be required to implement this exact behavior in a TPM. In this work, we use the same core idea but slightly change the communication, such that we can leverage the flexible TPM.Commit and TPM.Sign commands and avoid introducing new TPM commands. In addition, we give the TPM all the input it requires to create the non-revocation proof, such that it does not need to keep any state between signing and creating the non-revocation proof. More precisely, we can construct the non-revocation proof based on our revised TPM interface using the Prove protocol. The host obtains $C_{i}$ and constructs $\pi_{\mathrm{SRL}, i} \leftarrow\left(C_{i}, \pi_{\mathrm{SRL}, i}^{\prime}\right)$ by running

$$
\begin{array}{r}
\left(C_{i}, \pi_{\mathrm{SRL}, i}^{\prime}\right) \leftarrow \operatorname{Prove}\left(h s k, 1_{\mathbb{G}_{1}}, 1|| b s n, 1, \perp, \gamma, 1 \| b s n_{i}, \perp,\right. \\
\left.\left\{\left(\gamma, 1 / \text { nym }_{1} 1 / \text { nym }_{i}, \perp\right)\right\}, \text { "sign" }, \perp\right),
\end{array}
$$

To verify $\pi_{\mathrm{SRL}, i}$ in the VERIFY algorithm, one parses $\pi_{\mathrm{SRL}, i}=\left(C_{i}, \pi_{\mathrm{SRL}, i}^{\prime}\right)$, checks that $C_{i} \neq 1_{\mathbb{G}_{1}}$, and verifies $\pi_{\mathrm{SRL}, i}^{\prime}$ w.r.t. $\left(C_{i}, 1 \| b s n_{i}\right.$, nym $_{i}$, nym $)$, where $\left(1 \| b s n_{i}\right.$, nym $\left._{i}\right) \in \mathrm{SRL}$.

Note that since signature-based revocation is independent of the concrete PBSign scheme used for the membership credential, the above proof instantiation and the revocation checks in VERIFY are the same for the $q$-SDH-based and LRSW-based schemes.

Concrete Instantiations. The description of the JOIN and SIGN protocols and the VERIFY and LINK algorithms are given in Fig. 4, using an abstract NIZK proof statement for $\pi_{\text {cred }}$, and a generic partially-blind signature scheme PBSign for obtaining the membership credential. The concrete instantiation for this proof depends on the instantiation used for the PBSign scheme. In the following two sections we describe how PBSign and $\pi_{\text {cred }}$ can be instantiated with a $q$ SDH-based scheme (BBS+ signature [18]) and a LRSW-based scheme (CL-signature [17]) respectively. The latter uses a novel way to blindly issue $\mathrm{CL}$ signatures, which is significantly more efficient than previous approaches and is of independent interest.

For both concrete instantiations we assume the availability of system parameters consisting of a security parameter $\tau$, a bilinear group $\mathbb{G}_{1}, \mathbb{G}_{2}, \mathbb{G}_{T}$ of prime order $p$ with generators $g_{1}$ of $\mathbb{G}_{1}$ and $g_{2}$ of $\mathbb{G}_{2}$ and bilinear map $e$, generated w.r.t $\tau$, and with $\bar{g}$ denoting the fixed generator used by the TPMs. Note that we will not repeat the parts of the DAA protocol that are independent of the PBSign instantiation, such as the signaturebased revocation, the revocation checks within VERIFY, and the LINK protocol.

\subsection{1 q-SDH-based DAA Instantiation}

Our $q$-SDH-based scheme is most similar to the scheme by Camenisch et al. [10], which in turn propose a provably secure version of the scheme by Brickell and $\mathrm{Li}$ [3], which is standardized as mechanism 3 in ISO/IEC 20008-2 [31]. In addition, their and our scheme support membership credentials with selective attribute disclosure, similar to DAA with Attributes as proposed by Chen and Urian [32].

We now show how to instantiate PBSign and the affected proofs with $q$-SDH-based BBS+ signatures yielding a provably secure $q$-SDH-based DAA scheme $\Pi_{\mathrm{qSDH}-\mathrm{DAA}}$ using the revised TPM 2.0 interfaces proposed in Sec. 4.

SETUP: The issuer generates its key pair $(i p k, i s k)$ as follows:

- Choose $\left(h_{0}, \ldots, h_{L}\right) \leftarrow \mathbb{G}_{1}^{L+1}, x \leftarrow \mathbb{Z}_{p}$, set $X \leftarrow g_{2}^{x}$ and $X^{\prime} \leftarrow g_{1}^{x}$, and prove $\pi_{i p k} \leftarrow \operatorname{SPK}\left\{x: X=g_{2}^{x} \wedge X^{\prime}=\right.$ $\left.g_{1}^{x}\right\}$ ("setup").

- Set $i p k \leftarrow\left(h_{0}, \ldots, h_{L}, X, X^{\prime}, \pi_{i p k}\right)$, and $i s k \leftarrow x$.

Protocol participants, when retrieving $i p k$, will verify $\pi_{i p k}$.

JOIN: Here we show how the host obtains the proof $\pi_{t p k}$ from the TPM and how the issuer computes the membership credential using the BBS+ signature scheme. For this scheme, 
we set $\tilde{g}=\bar{g}$, so $t p k=t p k^{\prime}$ and we can simplify $\pi_{t p k}$ to $\pi_{t p k} \leftarrow \mathrm{SPK}^{*}\left\{t s k: t p k=\bar{g}^{t s k}\right\}($ "join", $n)$.

- The host obtains $\pi_{t p k}$ by calling

$\left(*, \pi_{t p k}\right) \leftarrow \operatorname{Prove}(0, t p k, \perp, 1, \perp, 1, \perp, \perp, \emptyset, \perp,($ "join",$n))$.

- The issuer computes the membership credential cred $\leftarrow$ PBSign(isk,gpk, attrs) on the joint public key gpk and a set of attributes attrs $=\left(a_{1}, \ldots, a_{L}\right)$ with $i s k=x$ as follows: It chooses a random $(e, s) \in \mathbb{Z}_{p}^{2}$, and derives

$$
A \leftarrow\left(g_{1} \cdot h_{0}^{s} \cdot g p k \cdot \prod_{i=1}^{L} h_{i}^{a_{i}}\right)^{\frac{1}{e+x}} .
$$

That is, the issuer creates a standard BBS+ signature on the message $\left(g s k, a_{1}, \ldots, a_{L}\right)$, where $g s k=t s k+h s k$ is blindly signed in form of $g p k=\bar{g}^{g s k}$. It sets cred $\leftarrow(A, e, s)$.

- The host upon receiving (cred,attrs) from the issuer, computes $b \leftarrow g_{1} \cdot h_{0}^{s} \cdot g p k \cdot \prod_{i=1}^{L} h_{i}^{a_{i}}$, and checks that $e\left(A, X g_{2}^{e}\right)=e\left(b, g_{2}\right)$. Finally, it sets $\operatorname{cred}^{\prime} \leftarrow((A, e, s), b)$.

SIGN: A platform holding a membership credential cred $^{\prime}=$ $\overline{((A, e}, s), b)$ on platform key $g s k$ and attributes attrs can sign message $m$ w.r.t. basename $b s n$, attribute disclosure $(D, I)$, and signature-based revocation list SRL. As shown in Fig. 4, each signature $\sigma$ contains a proof of a membership credential $\pi_{\text {cred }}$ w.r.t. the pseudonym nym $=\mathrm{H}_{\mathbb{G}_{1}}(1|| b s n)^{g s k}$, which are computed as follows:

- The host first randomizes the BBS+ credential $((A, e, s), b)$ : Choose $r_{1} \leftarrow \mathbb{Z}_{p}^{*}, r_{2} \leftarrow \mathbb{Z}_{p}, r_{3} \leftarrow \frac{1}{r_{1}}$, set $A^{\prime} \leftarrow A^{r_{1}}, \bar{A} \leftarrow$ $A^{\prime-e} \cdot b^{r_{1}}\left(=A^{\prime x}\right), b^{\prime} \leftarrow b^{r_{1}} \cdot h_{0}^{-r_{2}}$, and $s^{\prime} \leftarrow s-r_{2} \cdot r_{3}$. The host and TPM then jointly compute the following proof $\pi_{\text {cred }}^{\prime}$. We denote by $\bar{D}=\{1, \ldots, L\} \backslash D$ the indices of attributes that are not disclosed.

$$
\begin{gathered}
\pi_{c r e d}^{\prime} \leftarrow \mathrm{SPK}^{*}\left\{\left(g s k,\left\{a_{i}\right\}_{i \in \bar{D}}, e, r_{2}, r_{3}, s^{\prime}\right):\right. \\
g_{1}^{-1} \prod_{i \in D} h_{i}{ }^{-a_{i}}=b^{\prime-r_{3}} h_{0}^{s^{\prime}} \bar{g}^{g s k} \prod_{i \in \bar{D}} h_{i}{ }^{a_{i}} \wedge \\
n y m=\mathrm{H}_{\mathbb{G}_{1}}(1 \| b s n)^{g s k} \wedge \\
\left.\bar{A} / b^{\prime}=A^{\prime-e} \cdot h_{0}^{r_{2}}\right\}((" s i g n ",(D, I), \mathrm{SRL}), m)
\end{gathered}
$$

This proof and pseudonym are computed by running

$$
\left(\text { nym }, \pi_{\text {cred }}^{\prime}\right) \leftarrow \operatorname{Prove}(h s k, d, \perp, 1, \perp, 1,1 \| b s n,
$$

$$
\left.\bar{A} / b^{\prime}, S,(\text { "sign", }(D, I), \mathrm{SRL}), m\right) \text {, }
$$

with $d \leftarrow g_{1}^{-1} \prod_{i \in D} h_{i}^{-a_{i}}$ and the set of all witnesses for the proof: $S \leftarrow$ $\left\{\left(-e, 1_{\mathbb{G}_{1}}, 1_{\mathbb{G}_{1}}, A^{\prime}\right),\left(r_{2}, 1_{\mathbb{G}_{1}}, 1_{\mathbb{G}_{1}}, h_{0}\right),\left(-r_{3}, b^{\prime}, 1_{\mathbb{G}_{1}}, 1_{\mathbb{G}_{1}}\right)\right.$, $\left.\left(s^{\prime}, h_{0}, 1_{\mathbb{G}_{1}}, 1_{\mathbb{G}_{1}}\right)\right\} \cup\left\{\left(a_{i}, h_{i}, 1_{\mathbb{G}_{1}}, 1_{\mathbb{G}_{1}}\right)\right\}_{i \in \bar{D}}$. The host then sets $\pi_{\text {cred }} \leftarrow\left(\bar{A}, A^{\prime}, b^{\prime}, \pi_{\text {cred }}^{\prime}\right)$.

VERIFY: To verify $\pi_{\text {cred }}=\left(\bar{A}, A^{\prime}, b^{\prime}, \pi_{\text {cred }}^{\prime}\right)$ w.r.t. $(i p k, \sigma, m, b s n,(D, I), \mathrm{RL}, \mathrm{SRL})$ and nym, parse $i p k=$ $\left(h_{0}, \ldots, h_{L}, X, X^{\prime}, \pi_{i p k}\right)$, check that $A^{\prime} \neq 1_{\mathbb{G}_{1}}$ and $e\left(A^{\prime}, X\right)=e\left(\bar{A}, g_{2}\right)$, and verify $\pi_{\text {cred }}^{\prime}$ with respect to message $m$, basename $b s n$, attribute disclosure $(D, I)$, signature revocation list $\mathrm{SRL}$, randomized credential $\left(\bar{A}, A^{\prime}, b^{\prime}\right)$, and pseudonym nym.

\subsubsection{LRSW-based DAA Instantiation}

We now demonstrate that an LRSW-based DAA scheme can be built on top of the new TPM interface. Our scheme is similar to the scheme by Chen, Page, and Smart [23], standardized as mechanism 4 of ISO/IEC 20008-2 [31], but includes the fixes to flaws pointed out by Bernhard et al. [33] and Camenisch et al. [9].

Note, for the sake of efficiency we do not include attributes in this scheme. Selective attribute disclosure can be supported using the extension by Chen and Urian [32], but it comes with a significant loss in efficiency. When attributes are required, the $q$-SDH-based scheme should be used.

A New Approach to Issue CL-Signatures. The main difference to the schemes by Bernhard et al. [33] and Camenisch et al. [9] is the way we prevent a static DH oracle when the membership credentials are generated. In LRSW-based schemes, cred is a CL-signature $(a, b, c, d)$ on $g s k$, where for blind signing the issuer chooses $\alpha \leftarrow \mathbb{Z}_{p}^{*}$ and sets

$$
a \leftarrow \bar{g}^{\alpha}, b \leftarrow a^{y}, c \leftarrow a^{x} \cdot g p k^{\alpha \cdot x y}, d \leftarrow g p k^{\alpha \cdot y},
$$

with $(x, y)$ denoting the issuer's signing key and $g p k=\bar{g}^{g s k}$ the platform public key. The DH oracle arises as the TPM must later prove knowledge of $d=b^{g s k}$, and $b$ is a value chosen by the issuer.

The schemes by Bernhard et al. [33] and Camenisch et al. [9] avoid such an oracle by letting the issuer prove $\pi \stackrel{\operatorname{SPK}}{ }\left\{(\alpha \cdot y): b=\bar{g}^{\alpha \cdot y} \wedge d=g p k^{\alpha \cdot y}\right\}$. Thus, the issuer proves that it correctly computed $d=b^{g s k}$, which shows the TPM that it can use $b$ as a generator without forming a static DH oracle (as the issuer already knows $d$ ). The TPM must therefore verify $\pi$, store $(b, d)$ along with its key, and only use these values in the subsequent SPKs.

While allowing for a security proof under the standard DL assumption, realizing this approach would require significant changes to the TPM interface to verify and store the additional key material. Further, the TPM 2.0 specification aimed to provide a generic interface for a number of protocols, and adding LRSW-DAA specific changes would thwart this effort.

Our goal is to keep the TPM protocol as generic and simple as possible, and we propose a novel and more elegant solution that avoids the DH oracle without requiring the TPM to verify a zero-knowledge proof. For the sake of simplicity we assume $g s k=t s k$ for the exposition of our core idea, and only include the split-key approach $g s k=t s k+h s k$ in the full protocol specification.

The issuer chooses a random nonce $n$ and we derive $b \leftarrow$ $\mathrm{H}_{\mathbb{G}_{1}}(0 \| n)$. The TPM receives $n$, derives $b$ and sends $d=b^{g s k}$ to the issuer. Note that $d$ does not leak information about gsk when we model $\mathrm{H}_{\mathbb{G}_{1}}$ as a random oracle. The issuer then completes the credential by computing

$$
a \leftarrow b^{1 / y}, \quad c \leftarrow(a \cdot d)^{x} .
$$

It is easy to see that the values $(a, b, c, d)$ derived in that way, form a standard CL signature on $g s k$ as in the existing schemes. Note that we now use $\mathrm{H}_{\mathbb{G}_{1}}$ in both the join protocol 
and to create pseudonyms while signing. We prefix the hash computation with a bit to distinguish these cases, to prevent losing privacy when signing with a basename bsn equal to nonce $n$.

This new blind issuance protocol is provably secure under the generalized LRSW assumption as introduced in Sec. 2, which we prove as one step in our full security proof in the full version of this paper. We need the generalized LRSW assumption, as the issuer already commits to values $a$ and $b$ before getting the $d$ value and computing $c$ based on $d$. One can easily modify the issuance scheme to be secure under the standard LRSW assumption though, one needs to prepend one extra round between the TPM and the issuer before running the issuance as described above. Therein, the issuer sends a nonce $n^{\prime}$ to the TPM, and the TPM responds with a proof $\pi \leftarrow \mathrm{SPK}^{*}\left\{g s k: g p k=\bar{g}^{g s k}\right\}\left(n^{\prime}\right)$. The issuer verifies $\pi$ and then continues with the issuance as described above. In the security proof this allows to extract $g s k$ from $\pi$ and we can obtain the full signature $(a, b, c)$ on $g s k$ from the LRSW oracle. Note that this extra round can be implemented with our revised TPM interface as well, but slightly reduces the efficiency of the overall JOIN protocol.

We now describe how this new issuance protocol is used in the LRSW-based instantiation of our DAA protocol. We denote the DAA protocol given in Fig. 4 instantiated with the LRSW-based membership credential and the proofs described below as $\Pi_{\mathrm{LRSW}}$-DAA.

SETUP: The issuer generates its key pair $(i p k, i s k)$ as follows:

- Choose $x, y \leftarrow \mathbb{Z}_{p}^{*}$, set $X \leftarrow g_{2}^{x}, Y \leftarrow g_{2}^{y}$, and compute $\pi_{i p k} \stackrel{\leftrightarrow}{\leftarrow} \operatorname{SPK}\left\{(x, y): X=g_{2}^{x} \wedge Y=g_{2}^{y}\right\}$ ("setup").

- Set $i p k \leftarrow\left(X, Y, \pi_{i p k}\right)$, and $i s k \leftarrow(x, y)$.

When first getting the issuer public key, protocol participants will check $Y \neq 1_{\mathbb{G}_{2}}$ and verify $\pi_{i p k}$.

JOIN: Opposed to the $q$-SDH-based protocol, we make use of the flexibility for the generator of the platform's key. That is, instead of using $\bar{g}$ we will use $\tilde{g}=\mathrm{H}_{\mathbb{G}_{1}}(0|| n)$ which will also serve as the $b$-value in the improved issuance of CL credentials as described above.

- First, upon receiving $n$ from the issuer, the host and TPM create $g p k, t p k^{\prime}, \pi_{t p k}, \pi_{g p k}$ based on $\tilde{g}=b=\mathrm{H}_{\mathbb{G}_{1}}(0 \| n)$. Recall that the TPM authenticates only the value $t p k=\bar{g}^{t s k}$, so the TPM must prove that $t p k^{\prime}=\tilde{g}^{t s k}$ uses the same $t s k$ as in its authenticated public key tpk:

$\pi_{t p k} \leftarrow \mathrm{SPK}^{*}\left\{t s k: t p k=\bar{g}^{t s k} \wedge t p k^{\prime}=\tilde{g}^{t s k}\right\}($ "join", $n)$

The TPM's key contribution $t p k^{\prime}$ and the proof $\pi_{t p k}$ are created via the Prove protocol for the following input:

$$
\begin{aligned}
\left(t p k^{\prime}, \pi_{t p k}\right) \leftarrow \operatorname{Prove}(0, t p k, \perp, 1, & \perp, 1,(0 \| n), \\
& \perp, \emptyset, \perp,(\text { "join", } n))
\end{aligned}
$$

The host then picks a key hsk, computes $g p k=t p k^{\prime}$. $\tilde{g}^{h s k}$ and $\pi_{g p k}$ (as described in Fig. 4) and finally sends $t p k, t p k^{\prime}, \pi_{t p k}, \pi_{g p k}, g p k$ to the issuer.
- Then, the issuer blindly completes the CL signature on $g s k=t s k+h s k$ as described above: the issuer computes $a \leftarrow \tilde{g}^{1 / y}, c \leftarrow(a \cdot g p k)^{x}$, and sets cred $\leftarrow(a, c)$. Note that $g p k=\tilde{g}^{g s k}=b^{g s k}$, so we can use this as the $d$-value of the credential.

- The host upon receiving cred $=(a, c)$ from the issuer verifies that $a \neq 1_{\mathbb{G}_{1}}, e(a, Y)=e\left(\tilde{g}, g_{2}\right)$, and $e\left(c, g_{2}\right)=$ $e(a \cdot g p k, X)$. Finally, the host sets $\operatorname{cred}^{\prime}=(a, \tilde{g}, c, g p k, n)$.

SIGN: We now describe how to instantiate the membership proof $\pi_{\text {cred }}$ for such CL signatures with our TPM methods.

- The host retrieves the join record $\left(h s k, c_{r e d}\right)$ and randomizes the CL credential cred $^{\prime}=(a, \tilde{g}, c, g p k, n)$ by $r \leftrightarrow \mathbb{Z}_{p}^{*}$ and setting $a^{\prime} \leftarrow a^{r}, \tilde{g}^{\prime} \leftarrow \tilde{g}^{r}, c^{\prime} \leftarrow c^{r}, g p k^{\prime} \leftarrow g p k^{r}$.

- The host and TPM then jointly compute nym $\leftarrow$ $\mathrm{H}_{\mathbb{G}_{1}}(1 \| b s n)^{g s k}$ for $g s k=t s k+h s k$ and prove knowledge of a CL credential on $g s k$ by creating:

$$
\begin{aligned}
& \pi_{\text {cred }}^{\prime} \leftarrow \mathrm{SPK}^{*}\left\{(g s k): g p k^{\prime}=\tilde{g}^{\prime g s k} \wedge\right. \\
& \left.n y m=\mathrm{H}_{\mathbb{G}_{1}}(1 \| b s n)^{g s k}\right\}((" s i g n ", \mathrm{SRL}), m)
\end{aligned}
$$

This proof and pseudonym nym are computed by

$$
\begin{array}{r}
\left(\text { nym }, \pi_{\text {cred }}^{\prime}\right) \leftarrow \operatorname{Prove}\left(h s k, g p k^{\prime},(0 \| n), r, \perp, 1,(1 \| b s n),\right. \\
\perp, \emptyset,(\text { "sign", SRL }), m) .
\end{array}
$$

Finally, the host sets $\pi_{\text {cred }} \leftarrow\left(a^{\prime}, \tilde{g}^{\prime}, c^{\prime}, g p k^{\prime}, \pi_{\text {cred }}^{\prime}\right)$.

VERIFY: To verify $\pi_{\text {cred }}=\left(a^{\prime}, \tilde{g}^{\prime}, c^{\prime}, g p k^{\prime}, \pi_{c r e d}^{\prime}\right)$ w.r.t. $(i p k, \sigma, m, b s n,(D, I), \mathrm{RL}, \mathrm{SRL})$ and nym, parse $i p k=$ $\left(X, Y, \pi_{i p k}\right)$, check that $a^{\prime} \neq 1_{\mathbb{G}_{1}}, e\left(a^{\prime}, Y\right)=e\left(\tilde{g}^{\prime}, g_{2}\right)$, and $e\left(c^{\prime}, g_{2}\right)=e\left(a^{\prime} \cdot g p k^{\prime}, X\right)$, and verify $\pi_{c r e d}^{\prime}$ with respect to ( $m$, bsn, SRL, $\tilde{g}^{\prime}, g p k^{\prime}$, nym).

\subsection{Security Properties of our Schemes}

In this section we informally discuss the security of our DAA schemes. For the formal security proof we refer to the full version of this paper.

Theorem 1 (Informal). Protocol $\Pi_{\mathrm{LRSW}-\mathrm{DAA}}$ is a secure anonymous attestation scheme under the Generalized LRSW and Decisional Diffie-Hellman assumptions in the random oracle model.

Theorem 2 (Informal). Protocol $\Pi_{\mathrm{qSDH}-\mathrm{DAA}}$ is a secure anonymous attestation scheme under the $q-S D H$ and Decisional Diffie-Hellman assumptions in the random oracle model.

The proofs of these two theorems are quite similar. In the following we give a proof sketch that treats both schemes at the same time, pointing out the differences when they arise.

Proof (Sketch). For each of the properties stated in Section 5.1, we argue why our schemes satisfy them. The actual security proof is structured quite differently as there we prove that an environment cannot distinguish between the interactions with the real world parties and with the ideal specification with a simulator. Nevertheless, the arguments presented here also appear in the full formal proof. 
Unforgeability. First, we argue that the adversary cannot use a credential from a platform with an honest TPM. In both our schemes, signatures are signature proofs of knowledge of the platform secret key $t s k+h s k$, as defined in (1). This means that from Lemma 2 we can directly conclude that the adversary cannot use the credential of a platform with an honest TPM. Second, the adversary cannot use a revoked credential on the key $g s k$ by a corrupt platform. For private-key based revocation, the platform proves that nym $=\mathrm{H}_{\mathbb{G}_{1}}(1 \| b s n)^{g s k}$ is correctly constructed, and the revocation check will reject signatures with that pseudonym. If signature-based revocation is used, a pair $\left(b s n_{i}, n y m_{i}=\mathrm{H}_{\mathbb{G}_{1}}(1 \| b s n)^{g s k}\right)$ is included in SRL. In proof $\pi_{\mathrm{SRL}, i}^{\prime}$, the adversary must prove that his $g s k$ is different than the one used in $n y m_{i}$, which contradicts the soundness of the zero knowledge proof.

It remains to show that the adversary cannot create signatures using a forged credential. For $\Pi_{q S D H-D A A}$, this clearly breaks the existential unforgeability of the BBS+ signature scheme, which is proven under the $q-\mathrm{SDH}$ assumption. For $\Pi_{\text {LRSW-DAA, }}$, we have to show that credentials are unforgeable under the generalized LRSW assumption. For this, we simulate the issuer with a generalized LRSW instance. When the join protocol starts, the issuer asks $\mathcal{O}_{X}^{\mathrm{a}, \mathrm{b}}$ for $(a, b)$. It chooses a fresh nonce $n$ and programs the random oracle $\mathrm{H}_{\mathbb{G}_{1}}(0 \| n)=b$. When it receives proofs $\pi_{t p k}, \pi_{g p k}$ it extracts $t s k$ and $h s k$ and sets $g s k=t s k+h s k$. It then calls $\mathcal{O}_{X, Y}^{c}$ on $g s k$ to complete the credential. Now, when the adversary creates a signature with a forged credential, we can extract a credential $\left(a^{*}, b^{*}, c^{*}\right)$ on the fresh $g s k^{*}$ breaking the generalized LRSW assumption.

Non-Frameability. An honest platform cannot be framed, under the Discrete Logarithm (DL) assumption (which is implied by the assumptions we make). The host sets $g p k$ and $\tilde{g}$ based on given the DL instance, and must simulate $\pi_{g p k}$ as it does not know $h s k$ such that $g p k=t p k \cdot \tilde{g}^{h s k}$. When signing, the host also simulates the zero-knowledge proofs. Now, if an adversary creates a signature that links to a signature of the honest platform, it must prove knowledge of the discrete logarithm of gsk. We rewind to extract and break the DL assumption.

Strong Privacy. Our DAA schemes fulfill strong privacy, meaning that privacy is guaranteed as long as the host is honest, i.e., even when the TPM involved in the generation of an attestation is malicious. By Lemma 1, the proofs created together with a (malicious) TPM are zero knowledge. This means we can simulate these proofs without the adversary noticing the difference. Further, note that a platform key $g s k=$ $t s k+h s k$ is uniformly distributed over $\mathbb{Z}_{p}$ as the host picks $h s k$ uniformly at random from $\mathbb{Z}_{p}$. To prove that signatures are unlinkable, we let honest hosts pick a fresh key gsk every time they sign with a new basename. This is indistinguishable using a hybrid argument, where in the $i$-th hop, we use a fresh key for $b s n_{i}$. Every hop is indistinguishable from the previous one under the Decisional Diffie-Hellman (DDH) assumption.

In a nutshell, the latter is proved as follows. Upon receiving a DDH instance $(\alpha, \beta, \gamma)$, program the random oracle so that
$\mathrm{H}_{\mathbb{G}_{1}}\left(1 \| b s n_{i}\right) \leftarrow \beta$. The host sets $\alpha$ as the $g p k$ value and simulates proof $\pi_{g p k}$. When signing, the host simulates the proof of knowledge and sets nym $\leftarrow \gamma$. If the DDH instance is a DDH tuple, the same key was used to sign, and if it is not a DDH tuple, a fresh key was used.

Signatures are now done using a fresh key for each basename and the proofs are simulated, therefore no adversary can possibly break the anonymity of signatures.

\section{DAA WITH FORWARD ANONYMITY}

An important reason to remove the DH oracle in the TPM interfaces is that such an oracle prevents forward anonymity. As $\mathrm{Xi}$ et al. [8] point out, a host that becomes corrupted can test whether signatures were generated by the embedded TPM using the $\mathrm{DH}$ oracle.

Modeling the property of forward anonymity requires one to consider adaptive corruptions, i.e., a signature made by a host should remain anonymous even when at some later point the host becomes corrupted. A property-based notion for this was formally introduced by $\mathrm{Xi}$ et al. [8]. However, extending our ideal specification to also provide this property is nontrivial. First, to enable forward anonymity, the DAA scheme must allow one to create signatures w.r.t. no basename, i.e., $b s n=\perp$ and forward anonymity only holds for such signatures. Otherwise, a host that becomes corrupt could trivially link previous signatures generated for some basename $b s n \neq \perp$, by simply requesting a new signature w.r.t. $b s n$ and test for relation via the link algorithm. This means we would have to remove signature-based revocation from our security model. Second, our formal security proof considers static corruptions, whereas forward anonymity is inherently about dynamic corruptions. Indeed, realizing a scheme secure w.r.t. dynamic corruptions would be much less efficient than the scheme we present in this paper.

Despite this, the TPM interfaces we define allow one to build a DAA scheme with forward anonymity (however, the other security properties hold only in presence of static corruptions). That is, if we remove signature-based revocation from our DAA protocols, they fulfill the notion of forward security by Xi et al. For LRSW-based DAA, signing with $b s n=\perp$ means that nym is omitted from the signature and proof $\pi_{\text {cred }}$. For $q$-SDH-based DAA, if $b s n=\perp$ then nym is replaced by $j^{g s k}$, where $j$ is taken uniformly at random from $\mathbb{G}_{1}$ by the TPM, as in the $q$-SDH-based scheme by Brickell and $\mathrm{Li}$ [3].

Proving the resulting scheme to be forward anonymous would work as follows. The forward anonymity game considers a corrupt issuer. This means $\mathcal{A}$ can instruct platforms to join, and $\mathcal{A}$ runs the issuer side of the protocol. $\mathcal{A}$ can request complete signatures from joined platforms. Next, $\mathcal{A}$ submits the identities of two platforms and a message. The challenger chooses one of the two platforms at random and returns a signature on the given message with basename bsn $=\perp$ on behalf of the chosen platform. The game now models the fact that the host becomes corrupted by giving $\mathcal{A}$ access to the 
TPM commands of the platforms, and $\mathcal{A}$ 's task is to find out which of the two platforms created the signature.

For both schemes, we can prove forward anonymity under the DDH assumption, using a similar proof strategy as for strong privacy. First, the challenger answers all oracles correctly. Next, we modify the game slightly. The challenge signature is now computed under a fresh key, instead of the key of one of the two platforms that $\mathcal{A}$ submitted. In this modified game, no adversary can win with probability better than $\frac{1}{2}$, as the bit that $\mathcal{A}$ has to guess is independent of $\mathcal{A}$ 's view. This means $\mathcal{A}$ can only have non-negligible advantage by distinguishing the two games. As argued in the strong privacy proof in Sect. 5.3, the modification in the games is unnoticeable under the DDH assumption. showing that our protocols without signature-based revocation satisfy forward anonymity under the DDH assumption.

\section{OTHER USES OF OUR TPM INTERFACES}

In many protocols, the user would like to store his keys in secure hardware rather than on a normal computer. This way, the keys are secure and some security is preserved as long as the trusted hardware is not compromised, even when the computer is compromised. This section shows that due to the generic design of our TPM interface, it can be used to secure the keys of other cryptographic protocols. As an example, we consider U-Prove and e-cash with keys stored in a TPM, such that an attacker cannot use a user's U-Prove credential or e-cash wallet without access to the TPM. We discuss these constructions here only informally, i.e., without providing a security proof, as a formal treatment would require a new security model and a detailed proof, which is beyond the scope of this paper. For ease of presentation, we place the full key in the TPM, although we could split the key over the TPM and host as in our DAA schemes.

\subsection{Device Bound U-Prove}

U-Prove [7] is an attribute-based credential system where credential issuance and credential presentation are unlinkable. In the issuance protocol, the user receives a credential with public key $h=\left(g_{0} g_{1}^{x_{1}} \ldots g_{n}^{x_{n}} g_{d}^{x_{d}}\right)^{\alpha}$, where $x_{1}, \ldots, x_{n}$ are the attribute values of the user, and $x_{d}$ is the device secret. The device secret makes sure that a secure device must be present to use the credential. To show the credential, the user must prove knowledge of $x_{1}, \ldots, x_{n}, x_{d}$, and $\alpha$ such that $g_{0}=g_{1}^{x_{1}} \ldots g_{n}^{x_{n}} g_{d}^{x_{d}} \cdot h^{-1 / \alpha}$, with the help of the secure device.

Our proposed changes for TPM 2.0 allow the TPM to be used as secure device for U-Prove. The value $x_{d}$ will be the TPM secret key, and generator $g_{d}$ must be the generator $\bar{g}$ known to the TPM. Then, the credential presentation proof $\mathrm{SPK}^{*}\left\{\left(x_{1}, \ldots, x_{n}, x_{d}, \alpha\right)\right.$ : $\left.g_{0}=g_{1}^{x_{1}} \ldots g_{n}^{x_{n}} g_{d}^{x_{d}} \cdot h^{-1 / \alpha}\right\}$ can be constructed by computing $($ nym,$\pi) \leftarrow \operatorname{Prove}\left(0, g_{0}, \perp, 1, \perp, 1, \perp, \perp\right.$, $\left.\left\{\left(a_{1}, g_{1}, \perp, \perp\right), \ldots,\left(a_{n}, g_{n}, \perp, \perp\right),(1 / \alpha, h, \perp, \perp)\right\}, \perp, \perp\right)$.

By Lemma 3, such proofs can only be made with a contribution from the TPM, so one's credentials cannot be stolen, unless the attacker can access the TPM.

\subsection{Compact E-Cash}

Compact E-Cash [11] allows users to withdraw coins from a bank, and later anonymously spend the coins. The protocol assumes that every user has a key pair $\left(s k_{U}, p k_{U}=g^{s k_{U}}\right)$ with which it can authenticate towards the bank. To withdraw $2^{l}$ coins, the user first authenticates towards the bank by proving knowledge of $s k_{U}$. The user picks wallet secrets $s, l$, where the bank adds randomness to $s$, and the bank places signature $\sigma$ on committed values $s k_{U}, s$, and $l$, using a CL signature. The result of the withdraw protocol is a wallet $\left(s k_{U}, s, t, \sigma, J\right)$, where $J$ is an $l$-bit counter.

To spend a coin at merchant $M$, the user computes $R \leftarrow$ $\mathrm{H}\left(p k_{M}\right.$, info $)$, where the merchant provides info. Next, the user computes a coin serial number $S \leftarrow g^{\frac{1}{s+J+1}}$ and value $T \leftarrow p k_{U} \cdot g^{\frac{R}{t+J+1}}$ which is used to detect double spending of coins. Finally, it proves

$$
\begin{aligned}
& \operatorname{SPK}\left\{\left(J, s k_{U}, s, t, \sigma\right): 0 \leq J<2^{l} \wedge S=g^{\frac{1}{s+J+1}} \wedge\right. \\
& \left.T \leftarrow p k_{U} \cdot g^{\frac{R}{t+J+1}} \wedge \operatorname{Ver}\left(p k_{B},\left(s k_{U}, s, t\right), \sigma\right)=1\right\}
\end{aligned}
$$

We can instantiate Compact E-Cash such that users can securely store their secret key $s k_{U}$ inside a TPM, using a trick similar as in our LRSW-based DAA scheme. To create its keys, the bank picks secret key $\left(x, y, z_{1}, z_{2}, z_{3}\right) \leftarrow \mathbb{Z}_{p}^{5}$ and sets public key $X \leftarrow g_{2}^{x}, Y \leftarrow g_{2}^{y}, Z_{1} \leftarrow g_{2}^{z_{1}}, Z_{2} \leftarrow g_{2}^{z_{2}}$, and $Z_{3} \leftarrow g_{2}^{z_{3}}$. The withdrawal of coins start by the bank picking a fresh nonce $n$, and sending $n, b \leftarrow \mathrm{H}(n), a \leftarrow b^{1 / y}, A_{i} \leftarrow a^{z_{i}}$ and $B_{i} \leftarrow b^{z_{i}}$ for $i=1,2,3$ to the user. The user authenticates by proving $p k_{U}=g_{1}^{s k_{U}} \wedge d=b^{s k_{U}}$, as in our LRSW-based DAA scheme. In addition, it picks $s^{\prime}, t$, and $r$, and commits to them using generators $B_{1}, B_{2}$, and $B_{3}: C \leftarrow B_{1}^{s^{\prime}} B_{2}^{t} B_{3}^{r}$. The user sends $C$ with a proof of knowledge of $\left(s^{\prime}, t, r\right)$ to the bank. The bank now adds randomness to $s^{\prime \prime}$ to $s^{\prime}$ by setting $C^{\prime} \leftarrow C \cdot B_{1}^{s^{\prime \prime}}$ and signs $s k_{U}, s=s^{\prime}+s^{\prime \prime}, t$, and $r$, by setting $c \leftarrow\left(a \cdot d \cdot C^{\prime y}\right)^{x}=a^{x+x y\left(m+z_{1} s+z_{2} t+z_{3} r\right)}$. The user now has signature $\sigma=\left(a, A_{1}, A_{2}, A_{3}, b, B_{1}, B_{2}, B_{3}, c, d\right)$.

To spend a coin, the user must compute $R, S$, and $T$, and prove that everything is correctly computed, as described above. The TPM holding $s k_{U}$ is only involved in proving that $\sigma$ is a valid CL signature on $\left(s k_{U}, s, t, r\right)$. It randomizes the signature by taking $\rho \leftarrow \mathbb{Z}_{p}^{*}$ and setting $a^{\prime} \leftarrow a^{\rho}, A_{i}^{\prime} \leftarrow$ $A_{i}^{\rho}, b^{\prime} \leftarrow b^{\rho}, B_{i}^{\prime} \leftarrow B_{i}^{\rho}, c^{\prime} \leftarrow c^{\rho}$. To prove this randomized signature signs $\left(s k_{U}, s, t, r\right)$, the user creates the following proof:

$$
\begin{aligned}
& \mathrm{SPK}^{*}\left\{\left(s k_{U}, s, t, r\right): e\left(c^{\prime}, g_{2}\right) / e\left(a^{\prime}, X\right)=\right. \\
& \left.e\left(b^{\prime}, X\right)^{s k_{U}} e\left(B_{1}^{\prime}, X\right)^{s} e\left(B_{2}^{\prime}, X\right)^{t} e\left(B_{3}^{\prime}, X\right)^{r}\right\} .
\end{aligned}
$$

This proof can be created with the TPM using $(*, \pi) \leftarrow \operatorname{Prove}\left(0, e\left(c^{\prime}, g_{2}\right) / e\left(a^{\prime}, X\right), n, \rho, X, 1, \perp, \perp\right.$, $\left.\left\{\left(s, e\left(B_{1}^{\prime}, X\right), \perp, \perp\right),\left(t, e\left(B_{2}^{\prime}, X\right), \perp, \perp\right)\right\}, \perp, \perp\right)$. Now, by Lemma 3, a wallet can only be used if the attacker has access to the TPM holding $s k_{U}$.

\section{CONCLUSION}

The TPM is a widely deployed security chip that can be embedded in platforms such that the platform can, among 
other things, anonymously attest to a remote verifier that it is in a secure state. Unfortunately, the current TPM 2.0 specification for DAA contains several flaws: it contains a static DH oracle towards the host and attestations built on top of this interface cannot be proven to be unforgeable. Fixes proposed in the literature are either impossible to implement within the constraints of the TPM, limit the functionality of the TPM interface, or open a subliminal channel that allows a malicious TPM to embed information in attestations, harming the privacy of the host.

We presented a revised TPM 2.0 interface and a Prove protocol for the host that allows the platform to create provably secure signature proofs of knowledge. The interface does not contain a DH oracle, and a corrupt TPM cannot break the zero-knowledge property of the resulting proofs.

Using the Prove protocol, we constructed two provably secure DAA schemes, one based on the LRSW assumption and one on the $q$-SDH assumption, including DAA extensions featuring signature-based revocation and attributes. Furthermore, we have shown that our TPM interface supports DAA schemes with forward anonymity and can be used to protect keys for other cryptographic schemes, such as e-cash and UProve. These latter applications were only shown informally, it remains future work to formally treat these applications.

The Trusted Computing Group has already adopted some of our proposed changes and is currently reviewing the remaining ones. It is our aim to bring these improvements to all the existing attestation standards, such as EPID, ISO/IEC 200082 , and FIDO attestation, such that all implementations are provably secure and can make use of TPMs.

\section{Acknowledgments.}

The first, third, and fourth author have been supported by the European Research Council (ERC) under Grant \#321310 (PERCY).

\section{REFERENCES}

[1] E. Brickell, J. Camenisch, and L. Chen, "Direct anonymous attestation," in ACM CCS. ACM, 2004, pp. 132-145.

[2] Trusted Computing Group, "TPM main specification version 1.2," 2004

[3] E. Brickell and J. Li, "A pairing-based DAA scheme further reducing TPM resources," in Trust and Trustworthy Computing: TRUST 2010 Springer Berlin Heidelberg, 2010, pp. 181-195.

[4] Trusted Computing Group, "Trusted platform module library specification, family "2.0"," 2014.

[5] L. Chen and J. Li, "Flexible and scalable digital signatures in TPM 2.0," in ACM CCS. ACM, 2013, pp. 37-48.

[6] C. Schnorr, "Efficient signature generation by smart cards," J. Cryptology, vol. 4, no. 3, pp. 161-174, 1991.

[7] C. Paquin and G. Zaverucha, "U-prove cryptographic specification v1.1 (revision 3)," December 2013. [Online]. Available: https://www.microsoft.com/en-us/research/publication/u-provecryptographic-specification-v1-1-revision-3/

[8] L. Xi, K. Yang, Z. Zhang, and D. Feng, "DAA-related APIs in TPM 2.0 revisited," in Trust and Trustworthy Computing: TRUST 2014, Springer International Publishing, 2014, pp. 1-18.

[9] J. Camenisch, M. Drijvers, and A. Lehmann, "Universally composable direct anonymous attestation," in Public-Key Cryptography - PKC 2016, Springer Berlin Heidelberg, 2016, pp. 234-264.

[10] - "Anonymous attestation using the strong diffie hellman assumption revisited," in Trust and Trustworthy Computing: TRUST 2016, Springer International Publishing, 2016, pp. 1-20.
[11] J. Camenisch, S. Hohenberger, and A. Lysyanskaya, "Compact ECash," in Advances in Cryptology - EUROCRYPT 2005. Springer Berlin Heidelberg, 2005, pp. 302-321.

[12] S. D. Galbraith, K. G. Paterson, and N. P. Smart, "Pairings for cryptographers," Discrete Applied Mathematics, vol. 156, no. 16, pp. 3113 - 3121, 2008, applications of Algebra to Cryptography.

[13] P. S. L. M. Barreto and M. Naehrig, "Pairing-friendly elliptic curves of prime order," in SAC 2005, Springer Berlin Heidelberg, 2006, pp. 319-331.

[14] D. Boneh and X. Boyen, "Short signatures without random oracles and the SDH assumption in bilinear groups," Journal of Cryptology, vol. 21, no. 2, pp. 149-177, 2007.

[15] A. Lysyanskaya, R. Rivest, A. Sahai, and S. Wolf, "Pseudonym systems," in SAC 2000, ser. Lecture Notes in Computer Science, Springer Berlin Heidelberg, 2000, vol. 1758, pp. 184-199.

[16] V. Shoup, "Lower Bounds for Discrete Logarithms and Related Problems,' in Advances in Cryptology - EUROCRYPT '97. Springer Berlin Heidelberg, 1997, pp. 256-266.

[17] J. Camenisch and A. Lysyanskaya, "Signature schemes and anonymous credentials from bilinear maps," in Advances in Cryptology - CRYPTO 2004, ser. Springer Berlin Heidelberg, 2004, vol. 3152, pp. 56-72.

[18] M. H. Au, W. Susilo, and Y. Mu, "Constant-size dynamic k-TAA," in Security and Cryptography for Networks: 5th International Conference, SCN 2006, Springer Berlin Heidelberg, 2006, pp. 111-125.

[19] J. Camenisch and M. Stadler, "Efficient group signature schemes for large groups," in Advances in Cryptology - CRYPTO '97, ser. Springer Berlin Heidelberg, 1997, vol. 1294, pp. 410-424.

[20] J. Camenisch, A. Kiayias, and M. Yung, "On the portability of generalized schnorr proofs," in Advances in Cryptology - EUROCRYPT 2009, Springer Berlin Heidelberg, 2009, vol. 5479, pp. 425-442.

[21] A. Fiat and A. Shamir, "How to prove yourself: Practical solutions to identification and signature problems," in Advances in Cryptology - CRYPTO '86, ser. Springer Berlin Heidelberg, 1987, vol. 263, pp. 186-194.

[22] M. Bellare and P. Rogaway, "Random oracles are practical: A paradigm for designing efficient protocols," in ACM CCS, ACM, 1993, pp. 62-73.

[23] L. Chen, D. Page, and N. Smart, "On the design and implementation of an efficient DAA scheme," in Smart Card Research and Advanced Application, Springer Berlin Heidelberg, 2010, vol. 6035, pp. 223-237.

[24] J. Camenisch, M. Drijvers, and A. Lehmann, "Anonymous attestation with subverted tpms," Cryptology ePrint Archive, Report 2017/200, 2017, http://eprint.iacr.org/2017/200.

[25] T. Acar, L. Nguyen, and G. Zaverucha, "A TPM diffie-hellman oracle," Cryptology ePrint Archive, Report 2013/667, 2013, http://eprint.iacr.org/.

[26] D. R. L. Brown and R. P. Gallant, "The static diffie-hellman problem," Cryptology ePrint Archive, Report 2004/306, 2004, http://eprint.iacr.org/2004/306.

[27] J. H. Cheon, "Security analysis of the strong diffie-hellman problem," in Advances in Cryptology - EUROCRYPT 2006, 2006, pp. 1-11.

[28] E. Brickell and J. Li, "Enhanced privacy ID from bilinear pairing for hardware authentication and attestation," International Journal of Information Privacy, Security and Integrity, vol. 1, no. 1, pp. 3-33, 2011.

[29] — "Enhanced privacy ID: A direct anonymous attestation scheme with enhanced revocation capabilities," in Proceedings of the 2007 ACM Workshop on Privacy in Electronic Society New York, NY, USA: ACM, 2007, pp. 21-30.

[30] R. Canetti, "Universally composable security: A new paradigm for cryptographic protocols," Cryptology ePrint Archive, Report 2000/067, 2000, http://eprint.iacr.org/.

[31] International Organization for Standardization, "ISO/IEC 20008-2: Information technology - Security techniques - Anonymous digital signatures - Part 2: Mechanisms using a group public key," 2013.

[32] L. Chen and R. Urian, "DAA-A: Direct anonymous attestation with attributes," in Trust and Trustworthy Computing: TRUST 2015, Springer International Publishing, 2015, pp. 228-245.

[33] D. Bernhard, G. Fuchsbauer, E. Ghadafi, N. Smart, and B. Warinschi, "Anonymous attestation with user-controlled linkability," International Journal of Information Security, vol. 12, no. 3, pp. 219-249, 2013.

[34] J. Camenisch, M. Drijvers, A. Edgington, A. Lehmann, R. Lindemann, and R. Urian, "FIDO ECDAA algorithm, implementation draft," https://fidoalliance.org/specs/fido-uaf-v1.1-id-20170202/fidoecdaa-algorithm-v1.1-id-20170202.html. 


\section{APPENDIX A}

FORMAL SECURITY MODEL

This section introduces our formal security model of DAA, which is based on the definition by Camenisch et al. [9], [10], [24]. At the end of this section we also compare the captured privacy guarantees in the presence of subverted TPM with the existing privacy notions, and to optimal privacy [24] in particular.

\section{Al Universal Composability}

Our security definition has the form of an ideal functionality $\mathcal{F}_{\text {pdaat }}$ in the Universal Composability (UC) framework [30]. In UC, an environment $\mathcal{E}$ gives inputs to the protocol parties and receives their outputs. In the real world, honest parties execute the protocol, over a network controlled by an adversary $\mathcal{A}$, who can also communicate freely with the environment $\mathcal{E}$. In the ideal world, honest parties forward their inputs to the ideal functionality $\mathcal{F}$. The ideal functionality internally performs the defined task and generates outputs for the honest parties. As $\mathcal{F}$ performs the task at hand in an ideal fashion, i.e., $\mathcal{F}$ is secure by construction.

Informally, a protocol $\Pi$ is said to securely realize an ideal functionality $\mathcal{F}$ if the real world is as secure as the ideal world. To prove that statement one has to show that for every adversary $\mathcal{A}$ attacking the real world, there exists an ideal world attacker or simulator $\mathcal{S}$ that performs an equivalent attack on the ideal world. More precisely, $\Pi$ securely realizes $\mathcal{F}$ if for every adversary $\mathcal{A}$, there exists a simulator $\mathcal{S}$ such that no environment $\mathcal{E}$ can distinguish the real world (with $\Pi$ and $\mathcal{A}$ ) from the ideal world (with $\mathcal{F}$ and $\mathcal{S}$ ).

\section{A2 Session Identifiers and Input/Output}

In the UC model, different instances of the protocol are distinguished with session identifiers. Here we use session identifiers of the form sid $=\left(\mathcal{I}\right.$, sid $\left.^{\prime}\right)$ for some issuer $\mathcal{I}$ and a unique string $s i d^{\prime}$. To allow several sub-sessions for the join and sign related interfaces we use unique sub-session identifiers $j$ sid and ssid.

Every party can give different inputs to the protocol. We distinguish these by adding different labels to these inputs, e.g., the host can give an input labeled with JOIN to request to join, and an input labeled with SIGN to start signing a message. Outputs are labeled in a similar way.

\section{A3 Ideal Functionality $\mathcal{F}_{\text {pdaa }}+$}

This section formally introduces our ideal DAA functionality $\mathcal{F}_{\text {pdaa }}$, which defines DAA with attributes, signaturebased revocation, and strong privacy. It is based on $\mathcal{F}_{\text {pdaa }}$ and $\mathcal{F}_{\text {daa }}^{l}$ by Camenisch et al. [10], [24]. We now give an informal overview of the interfaces of $\mathcal{F}_{\text {pdaa }}$, and present the full definition in Fig. 5.

Setup. The SETUP interface on input sid $=\left(\mathcal{I}\right.$, sid $\left.^{\prime}\right)$ initiates a new session for the issuer $\mathcal{I}$ and expects the adversary to provide algorithms (ukgen, sig, ver, link, identify) that will be used inside the functionality. ukgen creates a new key gsk and a tracing trapdoor $\tau$ that allows $\mathcal{F}_{\text {pdaa }}$ to trace signatures generated with $g s k$. sig, ver, and link are used by $\mathcal{F}_{\text {pdaa }}$ to create, verify, and link signatures, respectively. Finally, identify allows to verify whether a signature belongs to a certain tracing trapdoor. This allows $\mathcal{F}_{\text {pdaa }}$ to perform multiple consistency checks and enforce the desired nonframeability and unforgeability properties.

Note that the ver and link algorithms assist the functionality only for signatures that are not generated by $\mathcal{F}_{\text {pdaa }}+$ itself. For signatures generated by the functionality, $\mathcal{F}_{\text {pdaa }}+$ will enforce correct verification and linkage using its internal records. While ukgen and sig are probabilistic algorithms, the other ones are required to be deterministic. The link algorithm also has to be symmetric, i.e., for all inputs it must hold that $\operatorname{link}\left(\sigma, m, \sigma^{\prime}, m^{\prime}, b s n\right) \leftrightarrow \operatorname{link}\left(\sigma^{\prime}, m^{\prime}, \sigma, m, b s n\right)$.

Join. A host $\mathcal{H}_{j}$ can request to join with a TPM $\mathcal{M}_{i}$ using the JOIN interface. The issuer is asked to approve the join request, and choose the platform's attributes. $\mathcal{F}_{\text {pdaa }+}$ is parametrized by $L$ and $\left\{\mathbb{A}_{i}\right\}_{0<i \leq L}$, that offer support for attributes. $L$ is the amount of attributes every credential contains and $\mathbb{A}_{i}$ the set from which the $i$-th attribute is taken. When the issuer approves with attributes attrs $\in \mathbb{A}_{1} \times \ldots \times \mathbb{A}_{L}$, the functionality stores an internal membership record for $\mathcal{M}_{i}, \mathcal{H}_{j}$, attrs in Members indicating that from now on that platform is allowed to create attestations.

If the host is corrupt, the adversary must provide $\mathcal{F}_{\text {pdaa }}+$ with a tracing trapdoor $\tau$. This value is stored along in the membership record and allows the functionality to check via the identify function whether signatures were created by this platform. $\mathcal{F}_{\text {pdaa }}+$ uses these checks to ensure nonframeability and unforgeability whenever it creates or verifies signatures. To ensure that the adversary cannot provide bad trapdoors that would break the completeness or nonframeability properties, $\mathcal{F}_{\text {pdaa }}+$ checks the legitimacy of $\tau$ via the "macro" function CheckTtdCorrupt. This function checks that for all previously generated or verified signatures for which $\mathcal{F}_{\text {pdaa }}$ has already seen another matching tracing trapdoor $\tau^{\prime} \neq \tau$, the new trapdoor $\tau$ is not identified as a matching key as well. CheckTtdCorrupt is defined as follows:

$$
\begin{gathered}
\text { CheckTtdCorrupt }(\tau)=\nexists(\sigma, m, b s n):( \\
(\langle\sigma, m, b s n, *, *\rangle \in \text { Signed } \vee \\
\langle\sigma, m, b s n, *, 1\rangle \in \text { VerResults }) \wedge \\
\exists \tau^{\prime}:\left(\tau \neq \tau^{\prime} \wedge\left(\left\langle *, *, \tau^{\prime}\right\rangle \in \text { Members } \vee\right.\right. \\
\left.\left\langle *, *, *, *, \tau^{\prime}\right\rangle \in \text { DomainKeys }\right) \wedge \\
\text { identify } \left.\left.(\sigma, m, b s n, \tau)=\text { identify }\left(\sigma, m, b s n, \tau^{\prime}\right)=1\right)\right)
\end{gathered}
$$

Sign. After joining, a host $\mathcal{H}_{j}$ can use the SIGN interface to request a signature on a message $m$ with respect to basename $b s n$ while proving a certain predicate $p$ holds for his attributes and proving that he is not revoked by signature revocation list SRL. The signature will only be created when the TPM $\mathcal{M}_{i}$ 
explicitly agrees to signing $m$, a join record for $\mathcal{M}_{i}, \mathcal{H}_{j}$, attrs in Members exists such that attrs satisfy $p$ (if the issuer is honest), and the platform is not revoked by SRL.

When a platform wants to sign message $m$ w.r.t. a fresh basename $b s n, \mathcal{F}_{\text {pdaa }}$ generates a new key gsk (and tracing trapdoor $\tau$ ) via ukgen and then signs $m$ with that key. The functionality also stores the fresh key $(g s k, \tau)$ together with $b s n$ in DomainKeys, and reuses the same key when the platform wishes to sign repeatedly under the same basename. Using fresh keys for every signature naturally enforces the desired privacy guarantees: the signature algorithm does not receive any identifying information as input, and thus the created signatures are guaranteed to be anonymous (or pseudonymous in case $b s n$ is reused).

To guarantee non-frameability and completeness, our functionality further checks that every freshly generated key, tracing trapdoor and signature does not falsely match with any existing signature or key. More precisely, $\mathcal{F}_{\text {pdaa }+}$ first uses the CheckTtdHonest macro to verify whether the new key does not match to any existing signature. CheckTtdHonest is defined as follows:

$$
\begin{aligned}
& \text { CheckTtdHonest }(\tau)= \\
& \forall\langle\sigma, m, b s n, \mathcal{M}, \mathcal{H}\rangle \in \text { Signed : identify }(\sigma, m, b s n, \tau)=0 \wedge \\
& \forall\langle\sigma, m, b s n, *, 1\rangle \in \operatorname{VerResults}: \operatorname{identify}(\sigma, m, b s n, \tau)=0
\end{aligned}
$$

Likewise, before outputting $\sigma$, the functionality checks that no one else already has a key which would match this newly generated signature.

Finally, for ensuring unforgeability, the signed message, basename, attribute predicate, signature revocation list, and platform identity are stored in Signed, which will be used when verifying signatures.

Verify. Signatures can be verified by any party using the VERIFY interface. $\mathcal{F}_{\text {pdaat }}$ uses its internal Signed, Members, and DomainKeys records to enforce unforgeability and non-frameability. It uses the tracing trapdoors $\tau$ stored in Members and DomainKeys to find out which platform created this signature. If no match is found and the issuer is honest, the signature is a forgery and rejected by $\mathcal{F}_{\text {pdaa }+ \text {. If }}$ the signature to be verified matches the tracing trapdoor of some platform with an honest host, but the signing records do not show that they signed this message w.r.t. the basename, attribute predicate, and signature revocation list, $\mathcal{F}_{\text {pdaa }}$ again considers this to be a forgery and rejects. If the platform has an honest TPM, only checks on the message and basename are made. If the records do not reveal any issues with the signature, $\mathcal{F}_{\text {pdaa }}$ uses the ver algorithm to obtain the final result.

The verify interface also supports verifier-local revocation. The verifier can input a revocation list RL containing tracing trapdoors, and signatures matching any of those trapdoors are no longer accepted.

Link. Using the LINK interface, any party can check whether two signatures $\left(\sigma, \sigma^{\prime}\right)$ on messages $\left(m, m^{\prime}\right)$ respectively, gen- erated with the same basename $b s n$ originate from the same platform or not. $\mathcal{F}_{\text {pdaa }}$ again uses the tracing trapdoors $\tau$ stored in Members and DomainKeys to check which platforms created the two signatures. If they are the same, $\mathcal{F}_{\text {pdaa }}$ outputs that they are linked. If it finds a platform that signed one, but not the other, it outputs that they are unlinked, which prevents framing of platforms with an honest host.

Conventions. The full definition of $\mathcal{F}_{\text {pdaat }}$ is presented in Fig. 5. We use a number of conventions to simplify the defini-

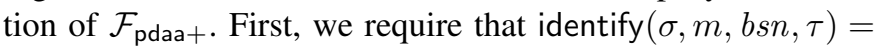
0 if $\sigma$ or $\tau$ is $\perp$. Second, whenever we need approval from the adversary to proceed, $\mathcal{F}_{\text {pdaa }}$ sends an output to the adversary and waits for a response. This means that in that join or sign session, no other inputs are accepted except the expected response from the adversary. Third, if any check that $\mathcal{F}_{\text {pdaa }}+$ makes fails, the sub-session is invalidated and $\perp$ is output to the caller.

\section{A4 Comparison of $\mathcal{F}_{\text {pdaa }}+$ with Previous Definitions}

Our functionality $\mathcal{F}_{\text {pdaa }}$ is based on previous UC-based DAA functionalities $\mathcal{F}_{\text {daa }}^{l}$ [9], $\mathcal{F}_{\text {daa }+}^{l}$ [10] which extends $\mathcal{F}_{\text {daa }}^{l}$ with attributes and signature-based revocation, and $\mathcal{F}_{\text {pdaa }}$ [24], which strengthens the privacy guarantees of $\mathcal{F}_{\text {daa }}^{l}$. We now show how our functionality compares to these other DAA functionalities.

Attributes and Signature-based Revocation. Our functionality $\mathcal{F}_{\text {pdaa }}$ supports adding attributes to the membership credentials, and selectively disclosing attributes when signing, as well as signature-based revocation. $\mathcal{F}_{\text {pdaa }}$ can be seen as $\mathcal{F}_{\text {pdaa }}$ extended with attributes and signature based revocations, in the same way that $\mathcal{F}_{\text {daa }+}^{l}$ adds these features to $\mathcal{F}_{\text {daa }}^{l}$.

Realistic TPM Interfaces. Contrary to the approach of $\mathcal{F}_{\text {daa }+}^{l}$, in our definition $\mathcal{F}_{\text {pdaa }+}$ the TPM is agnostic of attributes, predicates or the SRL. That is, when signing it neither explicitly sees or approves the attributes or SRL. This reflects that the actual TPM interfaces do not provide any such outputs or approvals either, and in fact, there is no practical reason to do so and would only make the TPM interfaces more complicated. Thus, we opted for adapting the functionality accordingly.

Similarly, the previous UC-based definitions [9], [10], [24] let the TPM approve both the message and basename for which the hosts requests as signature. In this definition, the TPM is only responsible for approving the message being signed, but does no longer receive (and approve) the basename. Again, this is done to better capture the actual TPM interfaces that provide such checks only for the message.

The resulting unforgeability and non-frameability guarantees are as follows. No adversary can sign a message $m$ w.r.t. basename $b s n$, attribute predicate $p$, and signature revocation list SRL, if the host did not sign exactly that. If the TPM is honest but the host is corrupt, the unforgeability is a bit weaker, as the TPM only checks the message. Therefore, if the TPM signed message $m$, the adversary is allowed to 
1) Issuer Setup. On input (SETUP, sid) from issuer $\mathcal{I}$.

- Verify that $\operatorname{sid}=\left(\mathcal{I}\right.$, sid $\left.^{\prime}\right)$.

- Output (SETUP, sid) to $\mathcal{A}$ and wait for input (ALG, sid, sig, ver, link, identify, ukgen) from $\mathcal{A}$.

- Check that ver, link, and identify are deterministic.

- Store (sid, sig, ver, link, identify, ukgen) and output (SETUPDONE, sid) to $\mathcal{I}$.

\section{Join}

2) Join Request. On input (JOIN, sid, jsid, $\mathcal{M}_{i}$ ) from host $\mathcal{H}_{j}$.

- Output (JOINSTART, sid, jsid, $\mathcal{M}_{i}, \mathcal{H}_{j}$ ) to $\mathcal{A}$ and wait for input (JOINSTART, sid, jsid) from $\mathcal{A}$.

- Create a join session record $\left\langle j s i d, \mathcal{M}_{i}, \mathcal{H}_{j}, \perp\right.$, status $\rangle$ with status $\leftarrow$ delivered.

- Abort if $\mathcal{I}$ is honest and a record $\left\langle\mathcal{M}_{i}, *, *\right\rangle \in$ Members already exists.

- Output (JOINPROCEED, sid, jsid, $\mathcal{M}_{i}$ ) to $\mathcal{I}$.

3) $\mathcal{I}$ Join Proceed. On input (JOINPROCEED, sid, jsid, attrs) from $\mathcal{I}$, with attrs $\in \mathbb{A}_{1} \times \ldots \times \mathbb{A}_{L}$.

- Output (JOINCOMPLETE, sid, jsid) to $\mathcal{A}$ and wait for input (JOINCOMPLETE, sid, jsid, $\tau$ ) from $\mathcal{A}$.

- Update the session record $\left\langle j s i d, \mathcal{M}_{i}, \mathcal{H}_{j}\right.$, status $\rangle$ with status $=$ delivered to complete.

- If $\mathcal{H}_{j}$ is honest, set $\tau \leftarrow \perp$.

- Else, verify that the provided tracing trapdoor $\tau$ is eligible by checking CheckTtdCorrupt $(\tau)=1$.

- Insert $\left\langle\mathcal{M}_{i}, \mathcal{H}_{j}, \tau\right.$, attrs $\rangle$ into Members and output (JOINED, sid, jsid, attrs) to $\mathcal{H}_{j}$.

\section{Sign}

4) Sign Request. On input (SIGN, sid, ssid, $\mathcal{M}_{i}, m, b s n, p$, SRL) from $\mathcal{H}_{j}$ with $p \in \mathbb{P}$.

- If $\mathcal{H}_{j}$ is honest and no entry $\left\langle\mathcal{M}_{i}, \mathcal{H}_{j}, *\right.$,attrs $\rangle$ with $p($ attrs $)=1$ exists in Members, abort.

- If $\mathcal{H}_{j}$ is corrupt, set $\sigma \leftarrow \perp$. If $\mathcal{H}_{j}$ is honest, generate the signature for a fresh or established key:

- Retrieve $(g s k, \tau) \quad$ from $\left\langle\mathcal{M}_{i}, \mathcal{H}_{j}, b s n, g s k, \tau\right\rangle \quad \in$ DomainKeys. If no such entry exists, set $(g s k, \tau) \leftarrow$ ukgen () , check CheckTtdHonest $(\tau)=1$, and store $\left\langle\mathcal{M}_{i}, \mathcal{H}_{j}\right.$, bsn, gsk, $\left.\tau\right\rangle$ in DomainKeys.

- Compute signature $\sigma \leftarrow \operatorname{sig}(g s k, m, b s n, p, \mathrm{SRL})$, check $\operatorname{ver}(\sigma, m, b s n, p, \mathrm{SRL})=1$.

- Check identify $(\sigma, m, b s n, \tau)=1$ and that there is no $\left(\mathcal{M}^{\prime}, \mathcal{H}^{\prime}\right) \neq\left(\mathcal{M}_{i}, \mathcal{H}_{j}\right)$ with tracing trapdoor $\tau^{\prime}$ registered in Members or DomainKeys with identify $\left(\sigma, m, b s n, \tau^{\prime}\right)=1$.

- Create a sign session record $\left\langle s s i d, \mathcal{M}_{i}, \mathcal{H}_{j}, m, b s n, p, \mathrm{SRL}, \sigma\right.$, status $\rangle$ with status $\leftarrow$ request.

- Output (SIGNPROCEED, sid, ssid, $m$ ) to $\mathcal{M}_{i}$ when it is honest, and (SIGNPROCEED, sid, ssid, $m, b s n$, SRL, $\sigma$ ) when
$\mathcal{M}_{i}$ is corrupt.

5) Sign Proceed. On input (SIGNPROCEED, sid, ssid) from $\mathcal{M}_{i}$.

- Look up record $\left\langle s s i d, \mathcal{M}_{i}, \mathcal{H}_{j}, m, b s n, p\right.$, SRL, $\sigma$, status $\rangle$ with status $=$ request and update it to status $\leftarrow$ complete.

- If $\mathcal{I}$ is honest, check that $\left\langle\mathcal{M}_{i}, \mathcal{H}_{j}, *\right.$, attrs $\rangle$ with $p($ attrs $)=1$ exists in Members.

- For every $\left(\sigma^{\prime}, m^{\prime}, b s n^{\prime}\right) \in \mathrm{SRL}$, find all $\left(\tau_{i}, \mathcal{M}_{i}^{\prime}, \mathcal{H}_{j}^{\prime}\right)$ from $\left\langle\mathcal{M}_{i}^{\prime}, \mathcal{H}_{j}^{\prime}, \tau_{i}, *\right\rangle \in$ Members and $\left\langle\mathcal{M}_{i}^{\prime}, \mathcal{H}_{j}^{\prime}, \tau_{i}\right\rangle \in$ DomainKeys where identify $\left(\sigma^{\prime}, m^{\prime}, b s n^{\prime}, *, \tau_{i}\right)=1$.

- Check that there are no two distinct $\tau$ values matching $\sigma^{\prime}$. - Check that no pair $\left(\tau_{i}, \mathcal{M}_{i}, \mathcal{H}_{j}\right)$ was found.

- Store $\left\langle\sigma, m, b s n, \mathcal{M}_{i}, \mathcal{H}_{j}, p, \mathrm{SRL}\right\rangle$ in Signed and output (SIGNATURE, sid, ssid, $\sigma$ ) to $\mathcal{H}_{j}$.

\section{Verify \& Link}

6) Verify. On input (VERIFY, sid, $m, b s n, \sigma, p, \mathrm{RL}, \mathrm{SRL})$ from some party $\mathcal{V}$.

- Retrieve all tuples $\left(\tau_{i}, \mathcal{M}_{i}, \mathcal{H}_{j}\right)$ from $\left\langle\mathcal{M}_{i}, \mathcal{H}_{j}, \tau_{i}, *\right\rangle \in$ Members and $\left\langle\mathcal{M}_{i}, \mathcal{H}_{j}, *, *, \tau_{i}\right\rangle \in$ DomainKeys where identify $\left(\sigma, m, b s n, \tau_{i}\right)=1$. Set $f \leftarrow 0$ if at least one of the following conditions hold:

- More than one $\tau_{i}$ was found.

- $\mathcal{I}$ is honest and no pair $\left(\tau_{i}, \mathcal{M}_{i}, \mathcal{H}_{j}\right)$ was found for which an entry $\left\langle\mathcal{M}_{i}, \mathcal{H}_{j}, *\right.$,attrs $\rangle \in$ Members exists with $p($ attrs $)=$ 1.

- $\mathcal{M}_{i}$ is honest but no entry $\left\langle *, m, b s n, \mathcal{M}_{i}, \mathcal{H}_{j}, *, *\right\rangle \in$ Signed exists.

- $\mathcal{H}_{j}$ is honest but no entry $\left\langle *, m, b s n, \mathcal{M}_{i}, \mathcal{H}_{j}, p, \mathrm{SRL}\right\rangle \in$ Signed exists.

- There is a $\tau^{\prime} \in \mathrm{RL}$ where identify $\left(\sigma, m, b s n, \tau^{\prime}\right)=1$ and no pair $\left(\tau_{i}, \mathcal{M}_{i}, \mathcal{H}_{j}\right)$ for an honest $\mathcal{H}_{j}$ was found.

- For some matching $\tau_{i}$ and $\left(\sigma^{\prime}, m^{\prime}, b s n^{\prime}\right) \in \mathrm{SRL}$, identify $\left(\sigma^{\prime}, m^{\prime}, b s n^{\prime}, \tau_{i}\right)=1$.

- If $f \neq 0$, set $f \leftarrow \operatorname{ver}(\sigma, m, b s n, p, \mathrm{SRL})$.

- Add $\langle\sigma, m, b s n, \mathrm{RL}, f\rangle$ to VerResults and output (VERIFIED, sid, $f$ ) to $\mathcal{V}$.

7) Link. On input (LINK, sid, $\left.\sigma, m, p, \mathrm{SRL}, \sigma^{\prime}, m^{\prime}, p^{\prime}, \mathrm{SRL}{ }^{\prime}, b s n\right)$ from a party $\mathcal{V}$

- Output $\perp$ to $\mathcal{V}$ if at least one signature $(\sigma, m, b s n, p, \mathrm{SRL})$ or $\left(\sigma^{\prime}, m^{\prime}, b s n, p^{\prime}, \mathrm{SRL}{ }^{\prime}\right)$ is not valid (verified via the VERIFY interface with $\mathrm{RL}=\emptyset$ ).

- For each $\tau_{i}$ in Members and DomainKeys compute $b_{i} \leftarrow$ identify $\left(\sigma, m, b s n, \tau_{i}\right)$ and $b_{i}^{\prime} \leftarrow$ identify $\left(\sigma^{\prime}, m^{\prime}, b s n, \tau_{i}\right)$ and do the following:

- Set $f \leftarrow 0$ if $b_{i} \neq b_{i}^{\prime}$ for some $i$.

- Set $f \leftarrow 1$ if $b_{i}=b_{i}^{\prime}=1$ for some $i$.

- If $f$ is not defined yet, set $f \leftarrow \operatorname{link}\left(\sigma, m, \sigma^{\prime}, m^{\prime}, b s n\right)$.

- Output (LINK, sid, $f$ ) to $\mathcal{V}$.

Fig. 5. Our ideal DAA functionality with strong privacy $\mathcal{F}_{\text {pdaa }}+$ 
create signatures on $m$ w.r.t. any $p$ and SRL that hold for the platform (i.e., the platform has the attributes to fulfill $p$ and is not revoked by SRL). The TPM does not explicitly approve $b s n$, but we force the (possibly corrupt) host to choose one bsn when signing, and signatures can only be valid if the message-basename combination was signed. Because the TPM does not explicitly approve the basename, our unforgeability with an honest TPM and corrupt host is slightly weaker than previous UC-based definitions [9], [10], [24] where the TPM must explicitly approve the basename.

When the host is honest but the TPM is corrupt, our definition also assures unforgeability and non-frameability like $\mathcal{F}_{\text {pdaa }}$, which provides stronger guarantees than [9] and [10], where both properties are not ensured when the TPM is corrupt.

Strong Privacy (vs. Optimal Privacy). Previous DAA schemes and definitions condition their privacy property on the honesty of the entire platform, i.e., as soon as either the TPM or host is corrupt, no privacy is guaranteed anymore. Whereas the honesty of the host is indeed necessary (a corrupt host can always break privacy by outputting identifying information), relying on the honesty of the TPM as well is an unnecessarily strong assumption. In fact, it even contradicts the original goal of DAA, namely to provide anonymous attestations without having to trust the hardware. This mismatch was recently discussed by Camenisch et al. [24] who propose the notion of DAA with optimal privacy which must hold even in the presence of corrupted or subverted TPMs. In contrast to $\mathcal{F}_{\text {daa }}^{l}$ and $\mathcal{F}_{\text {daa }+}^{l}$ where the adversary provides the signature whenever the host or TPM are corrupt, the functionality with optimal privacy $\mathcal{F}_{\text {pdaa }}$ outputs anonymous signatures as long as the host is honest. As the signatures are given directly to the host, the adversary learns nothing about them, even if the TPM is corrupt.

Unfortunately, the authors also show that optimal privacy cannot be achieved using constructions where the TPM and host together create a Fiat-Shamir proof of knowledge, which rules out the most efficient DAA schemes. The DAA protocol with optimal privacy proposed in [24] comes with a significant re-design, shifting most of the computations from the TPM to the host and would also require new operations to be implemented on the TPM.

The goal of this work is to obtain the best privacy properties with as minimal changes to the existing TPM and DAA specifications as possible. We therefore relax their notion of optimal privacy, and show how this can be achieved with modest modifications to the current DAA specifications and using our proposed TPM interfaces. Roughly, our new privacy notion - which we term strong privacy - allows the TPM to see the anonymous signature that is generated by the functionality and consequently also condition its behavior on the signature value. Thus, while the actual signature shown to the TPM is still guaranteed to be anonymous, the TPM can influence the final distribution of the signatures by blocking certain signature values (a signature is only output to the host when

\begin{tabular}{l|c|c|c}
\hline corrupt TPM & $\mathcal{F}_{\text {daa }}^{l}, \mathcal{F}_{\text {daa }+}^{l}$ & $\mathcal{F}_{\text {pdaa }+}$ (this work) & $\mathcal{F}_{\text {pdaa }}$ \\
\hline standard & - & - & + \\
isolated & - & + & ++ \\
\hline
\end{tabular}

Fig. 6. Comparison of privacy guarantees for an honest host in the presence of a corrupt TPM (either corrupt in the standard UC or isolated model of [24]).

the TPM explicitly approved it). A TPM performing such a "blocking attack" to alter the signature distribution can clearly be noticed by the host though, and thus, this attack has rather limited impact in practice.

The main reason why exposing the signature value to the TPM reduces the privacy guarantees stems from the way UC models corruption: In the standard UC corruption model, the adversary is allowed to see all inputs to the party he corrupts. That is, he will see the signatures given for approval to the TPM and can later re-identify the platform from the signature. However, as Camenisch et al. [24] argue, in case of the TPM this standard UC corruption model gives the adversary much more power than in reality. In the real world, the TPM is embedded inside a host who controls all communication with the outside world, i.e., the adversary cannot communicate directly with the TPM but only via the (honest) host. To model such subversion more accurately, [24] introduces isolated corruptions, where the adversary can specify the code that the isolated, yet subverted TPM will run, but cannot directly interact with the isolated TPM.

Applying this concept of isolated corruptions to our notion of strong privacy then yields significantly stronger privacy guarantees than with the standard corruption model: In signing the adversary no longer sees the signature which is only given to the isolated corrupt TPM. That is, when considering isolated TPM corruptions, the only difference to the optimal privacy notion of [24] is the aforementioned "blocking attack" which allows a corrupt TPM to influence the signature distribution, but with the risk of being caught by the host. Thus, w.r.t. isolated corruption, our notion of strong privacy is almost equivalent to optimal privacy, yet allows for significantly more efficient instantiation. An overview of the different privacy guarantees of this and the previous works is given in Fig. 6. 